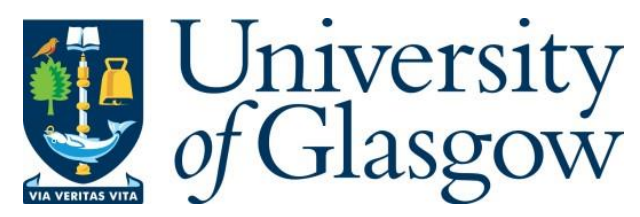

Mao, J., Zhang, L., Xiao, P. and Nikitopoulos, K. (2020) Interference analysis and power allocation in the presence of mixed numerologies. IEEE Transactions on Wireless Communications, (doi: 10.1109/TWC.2020.2990717).

There may be differences between this version and the published version. You are advised to consult the publisher's version if you wish to cite from it.

http://eprints.gla.ac.uk/214692/

Deposited on: 24 April 2020

Enlighten - Research publications by members of the University of Glasgow http://eprints.gla.ac.uk 


\title{
Interference Analysis and Power Allocation in the Presence of Mixed Numerologies
}

\author{
Juquan Mao, Lei Zhang, Senior Member, IEEE, Pei Xiao Senior Member, IEEE, \\ and Konstantinos Nikitopoulos Senior Member, IEEE,
}

\begin{abstract}
The flexibility in supporting heterogeneous services with vastly different technical requirements is one of the distinguishing characteristics of the fifth generation (5G) communication systems and beyond. One viable solution is to divide the system bandwidth into several bandwidth parts (BWPs), each having a distinct numerology optimized for a particular service. However, multiplexing of mixed numerologies over a unified physical infrastructure comes at the cost of induced interference. In this paper, we develop an analytical system model for inter-numerology interference (InterNI) analysis in orthogonal frequency-division multiplexing (OFDM) systems with and without filter processing in the presence of mixed numerologies. With the analytical model, the level of InterNI is quantified by the developed analytical metric, which is expressed as a function of several system parameters. This leads to an analysis and evaluation of these parameters for meeting a given distortion target. Moreover, a case study on power allocation utilizing the derived analysis is presented, where an optimization problem of maximizing the sum rate is formulated, and a solution is also provided. It is also demonstrated that a filtered-OFDM system better accommodates the coexistence of mixed numerologies. The proposed model provides an accurate analytical guidance for the multi-service design in 5G and beyond systems.
\end{abstract}

Index Terms-5G, mixed numerologies, OFDM, internumerology interference, multi-service, power allocation

\section{INTRODUCTION}

The fifth-generation $(5 \mathrm{G})$ mobile network is envisioned to support services with diversified requirements. The International Telecommunication Union (ITU) has categorized $5 \mathrm{G}$ services into three main usage scenarios [1]: enhanced mobile broadband (eMBB), ultra-reliable and low-latency communications (URLLC), and massive machine type communications (mMTC). Each of these scenarios demands distinct quality of service (QoS) requirements, such as throughput, latency, reliability, and the number of connected users. The coexistence of aforementioned services with diverse requirements poses challenges to legacy one-size-fits-all radio systems, such as the traditional 4G long term evolution (LTE) mobile networks, which were designed to meet requirements of the conventional mobile broadband (MBB) services with a single orthogonal

Manuscript received Jul 11, 2019; revised Dec 08, 2019. This work of J. Mao and P. Xiao was supported by the U.K. Engineering and Physical Sciences Research Council under Grant EP/R001588/1 and EP/S02476X/1.

J. Mao, P. Xiao and K. Nikitopoulos are with the Institute for Communication Systems (ICS), Home of 5G Innovation Centre, University of Surrey, Guildford, GU2 7XH, UK. (E-mail:\{juquan.mao, p.xiao, k.nikitopoulos\}@surrey.ac.uk)

L. Zhang is with the James Watt School of Engineering, University of Glasgow, Glasgow, G12 8QQ, UK. (E-Mail: lei.zhang@glasgow.ac.uk) frequency-division multiplexing (OFDM) numerology ${ }^{1}$. The one-size-fits-all structure may not be sufficiently flexible to meet all envisioned 5G use cases [3], [4]. For instance, an mMTC service might require smaller frequency subcarrier spacing (thus a longer symbol duration) to support massive delay-tolerant devices and to provide power boosting gains in some extreme cases; while vehicle-to-vehicle (V2V) communications necessitate significantly larger frequency subcarrier spacing (thus a smaller symbol duration) for stringent latency requirements and more robustness to Doppler spread.

Considering that the multitude of heterogeneous services must be provided simultaneously over a unified underlying physical layer, 5G new radio (NR) adopts a set of numerologies to suit different technical requirements and frequency bands [5]. The multiplexing of different numerologies can be implemented either in the time or the frequency domain. The latter has better compatibility and support for multi-service coexistence in comparison to the time domain counterpart [6]. However, multiplexing of different numerologies in the frequency domain inevitably comes at a cost of system performance degradation in terms of spectrum efficiency, scheduling flexibility, and computational complexity [7] due to the fact that the subcarrier orthogonality possessed by a single numerology no longer holds [8].

In the presence of mixed numerologies, subcarrier orthogonality maintains only within a numerology in an OFDM-based system. Subcarriers from one numerology interfere with those from others since subcarriers of one numerology may pick up leaked energy from subcarriers of other numerologies. The OFDM sinc-like transfer function decays with frequency $f$ as slow as $1 / f$ and substantial interference occurs between numerologies [6]. Additional signal processing on top of the legacy CP-OFDM waveform can suppress the interference leakage to achieve a defined emission mask and a good spectrum efficiency for mixed numerologies or asynchronous traffic.

Motivated by the aforementioned issues, various waveforms such as filter-bank multi-carrier (FBMC) [9], [10], generalized frequency-division multiplexing (GFDM) [11], windowed OFDM (W-OFDM) [12], [13], universal filtered multi-carrier (UFMC) [14], and filtered OFDM (f-OFDM) [15], [16], have been proposed. An overview and a comprehensive comparison

\footnotetext{
${ }^{1}$ Numerology refers to the configuration of waveform parameters, such as subcarrier spacing/symbol duration and cyclic prefix in OFDM [2]. Note: Although a typical setting for subcarrier spacing in LTE is $15 \mathrm{kHz}$, a subcarrier spacing of $7.5 \mathrm{kHz}$ is also defined for some common control channels and multicast/broadcast transmissions.
} 
among these waveforms in term of qualitative and quantitative analysis can be found in [2], [17], and [18]. Considering the performance-complexity trade-off, MIMO friendliness, and forward/backward compatibility, 5G NR [19] recommends to use spectral confinement techniques, such as filtering or windowing, to improve spectrum localization, provided that the techniques employed at the transmitter are transparent to the receiver. This implies that any additional signal processing on top of the cyclic prefix OFDM (CP-OFDM) waveform at the transmitter is not signaled to the receiver and thus generally unknown to the receiver. The feasibility of the transparent waveform processing is studied and confirmed in [20] by a simulation-based approach. However, it is not possible to have a better frequency localization without compromising the time localization according to the Heisenberg's principle [21]. When an OFDM signal is processed by filtering, it better localizes in the frequency domain but spreads in the time domain. In the case that the cyclic redundancy does not completely cover the signal time dispersion, the orthogonality among subcarriers within the same numerology is destroyed, giving rise to the intra-numerology interference (IntraNI) .

Interference analysis for multi-numerology systems has been attracting an increased interest recently. In particular, [22]-[24] study the factors contributing to interference in WOFDM systems. In [22], a system model for the interference analysis is established, and the analytical expression of InterNI power is derived as a function of several parameters in connection to the channel, guard band, and windowing. In [23] and [24], the authors investigate the impact of the guard interval in the time domain and the guard band in the frequency domain on the interference level, respectively, in order to improve bandwidth efficiency. A framework for subband filtered multi-carrier (SFMC) systems is introduced in [8], and the interference of UFMC systems is also analyzed in the presence of transceiver imperfections and insufficient guard interval between symbols. In [25], the authors develop a generic and universal optimization-based framework for fastconvolution-based f-OFDM (FC-f-OFDM) waveform processing for the $5 \mathrm{G}$ physical layer and evaluate its performance in scenarios following the test cases defined by 3GPP. In [26], a transparent spectral confinement approach is proposed for $\mathrm{f}$ OFDM/W-OFDM to enable multiplexing of multiple services with diverse requirements in one system band. In [27], the authors report a field trial conducted on a configurable testbed in a real-world environment for the performance evaluations of OFDM-based 5G waveform candidates, such as CP-OFDM, W-OFDM, and f-OFDM, and the field trial results confirm the feasibility of multi-numerology systems.

While both filtering and windowing enable frequency domain multiplexing of mixed numerologies, we focus on the filtering approach in this paper since it gives a better performance in terms of frequency localization and interference mitigation [17]. To the best of our knowledge, the analytical study conducted in this paper is the first of its kind accounting for all factors contributing to interference in f-OFDM systems. Such a study plays a pivotal role in providing guidance on system design for the coexistence of multi-service, enabling the development of efficient interference cancellation techniques, and facilitating the formulation of optimization problems for maximizing spectrum efficiency, etc. This motivates us to fill the gap by contributing the following:

- We first establish a generic analytical framework for OFDM/ f-OFDM systems to address the 5G NR numerology coexistence issue, in which the process of multiplexing different numerologies is formulated.

- Given the developed analytical model, the interference between different numerologies is analyzed, and the signal distortion level is quantified as a function of several system parameters. This allows us to study the impact of each of these parameters such that a more accurate and insightful approach is provided for system design in comparison to the simulation-based approach.

- Based on the developed analytical work, a case study on optimizing power allocation is presented, where an optimization problem of maximizing spectrum efficiency is formulated and solved in multi-numerology systems.

The reminders of the paper proceed as follows: Section II presents a generic OFDM/f-OFDM transceiver structure in the presence of mixed numerologies and describes the system model. Section III briefly discusses noise distribution and IntraNI, and Section IV focuses on deriving the analytical expression of the InterNI. A case study on optimizing power allocation in multi-numerology systems is provided in Section V. In Section VI, analytical and numerical results are presented. Finally, Section VII concludes the paper.

Notations: $\mathcal{E}\{\cdot\}$ denotes the expectation operator. We use boldface lower-case and upper-case characters to represent vectors and matrices, respectively. The operators $(\cdot)^{*},(\cdot)^{T}$ and $(\cdot)^{H}$ represent complex conjugate, transpose, and conjugate transpose, respectively. An $N$-dimensional identity matrix is denoted as $\mathbf{I}_{N}$. $\operatorname{diag}(\mathbf{x})$ returns a square diagonal matrix with the elements of vector $\mathbf{x}$ on its main diagonal, while $\operatorname{diag}(\mathbf{A})$ returns a column vector of the main diagonal elements of matrix $\mathrm{A}$. $\operatorname{blkdiag}(\mathbf{A}, n)$ returns a block diagonal matrix whose diagonal contains $n$ blocks of matrix $\mathbf{A} .|\mathbf{x}|$ returns a vector with each of its element being the magnitude of the corresponding element of $\mathbf{x} . \mathbb{B}, \mathbb{R}$, and $\mathbb{C}$ refers to binary, real, and complex space, respectively.

Numerology-related notations: $x^{(i)}, \mathbf{x}^{(i)}, \mathbf{X}^{(i)}$ indicate variable/vector/matrix only related to the $i$-th numerology. $\mathbf{x}_{k}^{(i)}$ represents a single-numerology signal in the $k$-th OFDM symbol of the $i$-th numerology. $\mathbf{x}_{k}^{<i>}$, angle brackets instead of parentheses, represents a mixed signal from all numerologies captured in the $k$-th OFDM symbol of the $i$-th numerology. $\mathbf{x}_{k}^{(i \leftarrow j)}$ indicates a signal from the $j$-th numerology captured in the $k$-th OFDM symbol of the $i$-th numerology.

\section{SYSTEM MODEL FOR GENERIC OFDM/F-OFDM SYSTEMS IN PRESENCE OF MIXED NUMEROLOGIES}

To enable the coexistence of mixed numerologies, the system bandwidth is divided into several bandwidth parts (BWPs) of arbitrary width, each with distinct numerology optimized for a particular service. For this reason, the two terms "BWP" and "numerology" are used interchangeably in 

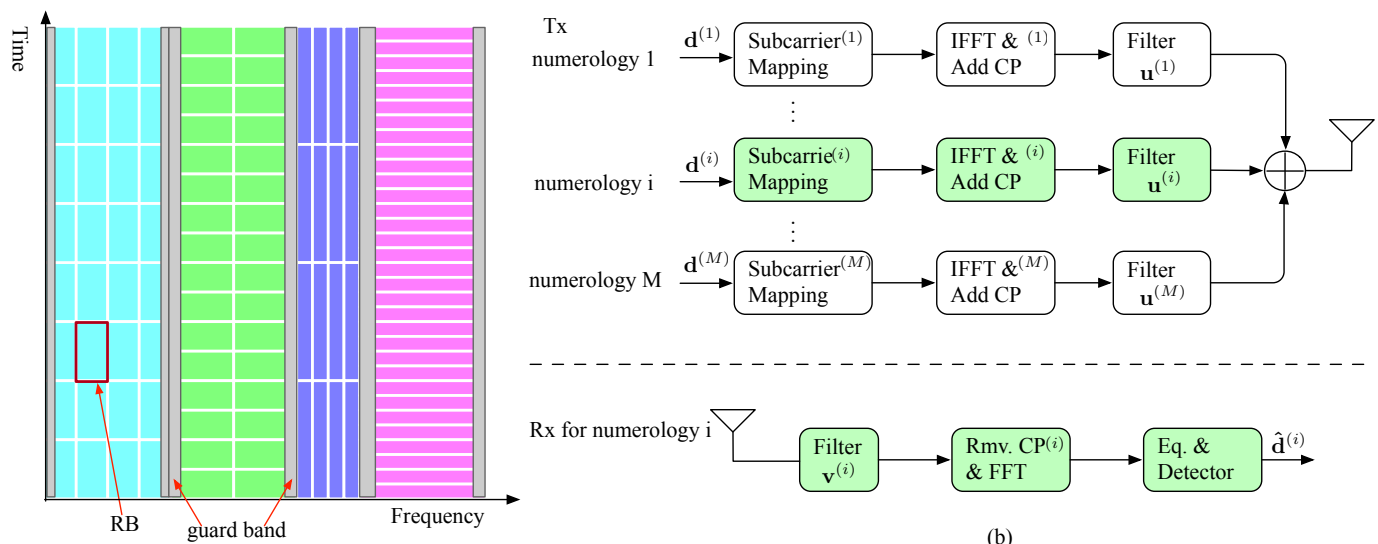

(a)

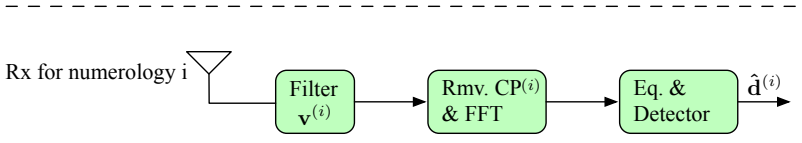

(b)

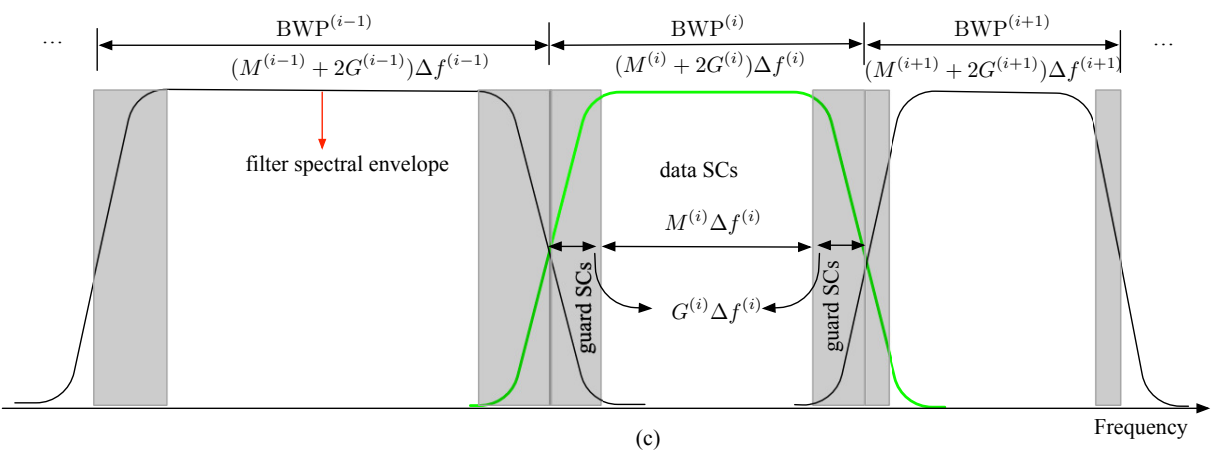

(c)

Fig. 1. System model of OFDM/f-OFDM in the presence of mixed numerologies, with $\mathbf{u}^{(i)}=1$ for OFDM. (a) An exemplary illustration of regular resource grid. (b) Downlink transceiver structure. (c) An exemplary illustration of multiplexing of mixed numerologies in the frequency domain. (RB stands for resource block, SC refers to subcarrier)

the paper whenever no ambiguity arises. Without loss of generality, one user per numerology is assumed. A communication system is considered to support a family of $M$ numerologies, $\mathcal{S}_{\text {num }}=\{1,2, \ldots, M\}$, which are related to each other via scaling, i.e.,

$$
\frac{\Delta f^{(i)}}{\Delta f^{(j)}}=\frac{\nu^{(i)}}{\nu^{(j)}}, \quad \frac{N_{\mathrm{cr}}^{(i)}}{N_{\mathrm{cr}}^{(j)}}=\frac{\nu^{(j)}}{\nu^{(i)}}
$$

where $\Delta f^{(i)}$ and $N_{\mathrm{cr}}^{(i)}$ denote subcarrier spacing and number of samples for cyclic redundancy in the $i$-th numerology. $\nu^{(i)} \in \mathbb{N}$ is a scaling factor which is chosen such that a subcarrier spacing is an integer and can be divided by all smaller ones, i.e.,

$$
\nu^{(i)}=2^{\mu^{(i)}}, \quad \forall i \in \mathcal{S}_{\text {num }},
$$

where $\mu^{(i)} \in\{0,1,2, \cdots\}$.

Assume that signal of different numerologies is processed with the same approach in the transceiver structure as depicted in Fig. 1 (b). For brevity, we only provide the detailed description of one numerology (the $i$-th numerology), which is also assumed as the victim of interest while signal from other numerologies serve as interference sources. Assume $N_{c}^{(i)}+2 G^{(i)}$ consecutive subcarriers in the range of $\tilde{\mathcal{M}}^{(i)}=$ $\left\{M_{0}^{(i)}-G^{(i)}, M_{0}^{(i)}-G^{(i)}+1, \ldots, M_{0}^{(i)}+N_{c}^{(i)}+G^{(i)}-1\right\}$ are assigned to the $i$-th BWP with a subcarrier spacing $\Delta f^{(i)}$ and a corresponding waveform shaping filter denoted as vector $\mathbf{u}^{(i)}$ in the time domain at the transmitter. $G^{(i)}<N_{c}^{(i)} / 2$ subcarriers from each side of the $i$-th BWP are reserved as guard subcarriers which do not carry any data symbols. For ease of reference, we denote the $N_{c}^{(i)}$ data bearing subcarriers in $\mathrm{BWP}^{(i)}$ as $\mathcal{M}^{(i)}=\left\{M_{0}^{(i)}, M_{0}^{(i)}+1, \ldots, M_{0}^{(i)}+N_{c}^{(i)}-1\right\}$. To avoid fractional subcarrier shifts, i.e., subcarrier frequencies should coincide with the natural grid of their numerology, guard bands are allocated inside BWPs [28], as illustrated in Fig. 1 (a) and (c). The guard band width between $\mathrm{BWP}^{(i)}$ and $\mathrm{BWP}^{(i+1)}$ is $G^{(i)} \Delta f^{(i)}+G^{(i+1)} \Delta f^{(i+1)}$.

Representing $K$ consecutive OFDM symbols in a sub-frame in the $i$-th numerology as an $N_{c}^{(i)} K$ dimensional vector yields

$$
\mathbf{d}^{(i)}=\left[\left(\mathbf{d}_{0}^{(i)}\right)^{T},\left(\mathbf{d}_{1}^{(i)}\right)^{T}, \cdots,\left(\mathbf{d}_{K-1}^{(i)}\right)^{T}\right]^{T},
$$

where the $k$-th OFDM symbol

$$
\mathbf{d}_{k}^{(i)}=\left[d_{k, 0}^{(i)}, d_{k, 1}^{(i)}, \cdots, d_{k, N_{c}^{(i)}-1}^{(i)}\right]^{T} \in \mathbb{C}^{N_{c}^{(i)} \times 1}
$$

with the individual element $d_{k, m}^{(i)}$ corresponding to the data symbol carried on the $m$-th subcarrier in the $k$-th OFDM symbol of the $i$-th numerology. The data symbols are assumed to be independent and identically distributed (i.i.d.) with $\mathcal{E}\left\{\mathbf{d}_{k}^{(i)}\left(\mathbf{d}_{k}^{(i)}\right)^{H}\right\}=\left(\sigma_{s}^{(i)}\right)^{2} \mathbf{I}$, where $\left(\sigma_{s}^{(i)}\right)^{2}$ is the average transmission power of QAM symbols for the $i$-th numerology.

The baseband transmitter and receiver procedures illustrated in Fig. 1 (b) are discussed in the sequel. It is worth mentioning that, although time domain filtering is adopted in our analysis 
framework, with respect to implementation, frequency domain multi-rate filtering is more efficient due to its low computational complexity [29].

\section{A. Transmitter Baseband Processing}

The transmitter baseband processing involves operations prior to transmission, which include OFDM modulation and filtering on each BWP and mixing signals from all the BWPs.

1) OFDM modulation: With respect to the $i$-th numerology, an $N^{(i)}$-point $\left(N^{(i)}=\frac{B}{\Delta_{f}^{(i)}} \geq N_{c}^{(i)}\right.$, where $B$ is the system bandwidth) inverse fast Fourier transform (IFFT) operation is performed on a per OFDM symbol basis, followed by the insertion of a cyclic redundancy (CR) of length $N_{\mathrm{cr}}^{(i)}$ for eliminating/mitigating inter-symbol interference (ISI). The CR is made up of two parts, cyclic prefix $(\mathrm{CP})$ of length $N_{\mathrm{cp}}^{(i)}$ and cyclic suffix $(\mathrm{CS})$ of length $N_{\mathrm{cs}}^{(i)}\left(N_{\mathrm{cr}}^{(i)}=N_{\mathrm{cp}}^{(i)}+N_{\mathrm{cs}}^{(i)}\right)$, with the former being adopted for combating forward ISI (fISI), and the latter for alleviating the backward ISI (b-ISI). To be compatible with CP-OFDM, CR can be implemented as an extended $\mathrm{CP}$ which incorporates $\mathrm{CP}$ and $\mathrm{CS}$ at the transmitter, and the fast Fourier transform (FFT) window is moved backward by the length of $\mathrm{CS}$ at the receiver. The $k$-th OFDM symbol can be expressed in the form of matrix multiplication as

$$
\mathbf{x}_{k}^{(i)}=\rho_{\mathrm{cr}}^{(i)} \mathbf{T}_{\mathrm{cr}}^{(i)} \mathbf{F}^{(i)} \mathbf{d}_{k}^{(i)} \in \mathbb{C}^{L^{(i)} \times 1},
$$

where $\mathbf{x}_{k}$ is a vector of dimension $L^{(i)}=N^{(i)}+N_{\mathrm{cr}}^{(i)} . \mathbf{F}^{(i)}$ is an $N^{(i)} \times N_{c}^{(i)}$ submatrix of the $N^{(i)}$-point IFFT matrix defined by its element on the $n$-th row and $m$-th column as $F_{n, m}^{(i)}=\sqrt{1 / N^{(i)}} \exp \left(\frac{j 2 \pi n\left(m+M_{0}^{(i)}\right)}{N^{(i)}}\right)$, and it is unitary since $\left(\mathbf{F}^{(i)}\right)^{H} \mathbf{F}^{(i)}=\mathbf{I}_{N_{c}^{(i)}} . \mathbf{T}_{\mathrm{cr}}^{(i)}=\left[\mathbf{I}_{\mathrm{cp}}^{(i)} ; \mathbf{I}_{N^{(i)}} ; \mathbf{I}_{\mathrm{cs}}^{(i)}\right]$ is an $L^{(i)} \times N^{(i)}$ dimensional CR insertion matrix, with $\mathbf{I}_{\mathrm{cp}}^{(i)}$ and $\mathbf{I}_{\mathrm{cs}}^{(i)}$ containing the last $N_{\mathrm{cp}}^{(i)}$ and the first $N_{\mathrm{cs}}^{(i)}$ rows of the identity matrix $\mathbf{I}_{N^{(i)}}$, respectively. $\rho_{\mathrm{cr}}^{(i)}=\sqrt{N^{(i)} / L^{(i)}}$ is the power normalization factor.

2) Transmitter filtering: The OFDM symbols are postprocessed by an appropriately designed spectrum shaping filter. The transmitted f-OFDM signal of the $i$-th numerology is finally obtained as

$$
\mathbf{s}^{(i)}=\mathbf{x}^{(i)} * \mathbf{u}^{(i)}
$$

where $\mathbf{x}^{(i)}=\left[\mathbf{x}_{0}^{(i)}, \mathbf{x}_{1}^{(i)}, \cdots, \mathbf{x}_{K-1}^{(i)}\right]^{T}$, and

$$
\mathbf{u}^{(i)}=\left[u_{0}^{(i)}, u_{1}^{(i)}, \cdots, u_{N_{u}^{(i)}}^{(i)}\right]^{T},
$$

is a length $N_{u}^{(i)}+1$ vector representing the impulse response of the transmitter filter $\left(\mathbf{u}^{(i)}=[1]\right.$ corresponds to the conventional OFDM). It is placed at the center of the assigned BWP and occupies the entire bandwidth. Filter length $N_{u}^{(i)}$ is chosen to be an even number to ensure the time domain symmetry, and less than or equal to half of the FFT size, i.e., $N_{u}^{(i)} \leq N^{(i)} / 2$, to restrict signal spreading within one OFDM symbol duration.
Fig. 2 illustrates how the IntraNI is introduced by filtering. As depicted in the figure, f-ISI/b-ISI is inflicted by the forward/backward spreading of the previous/next OFDM symbols to the current FFT window. The ICI (inter-carrier interference) of the current OFDM symbol comes from the energy loss due to its bi-directional spreading. To facilitate interference analysis, we derive the equivalent matrix form of a linear filtering process. The $L^{(i)}$ received samples relative to the $k$-th OFDM symbol are grouped into vector $\mathbf{s}_{k}^{(i)}$, thus obtaining

$$
\mathbf{s}_{k}^{(i)}=\mathbf{U}^{(i: u)} \mathbf{x}_{k-1}^{(i)}+\mathbf{U}^{(i: o)} \mathbf{x}_{k}^{(i)}+\mathbf{U}^{(i: l)} \mathbf{x}_{k+1}^{(i)},
$$

where the $L^{(i)} \times L^{(i)}$ forward spreading matrix $\mathbf{U}^{(i: u)}$ is a strictly upper triangular matrix with its $(r, c)$-th element being defined as

$$
\begin{aligned}
& U_{r, c}^{(i: u)}= \begin{cases}u^{(i)} & c \geq r+L^{(i)}-\frac{N_{u}^{(i)}}{2}+L^{(i)}+r-c \\
0, & c<r+L^{(i)}-\frac{N_{u}^{(i)}}{2}\end{cases} \\
& \left(0 \leq r, c \leq L^{(i)}-1\right) \text {. }
\end{aligned}
$$

The Toeplitz matrix $\mathbf{U}^{(i: o)}$ is specified by its first column

$$
\left[u_{\frac{N_{u}^{(i)}}{2}}^{(i)}, \cdots, u_{N_{u}^{(i)}}^{(i)}, \mathbf{0}_{1 \times\left(L^{(i)}-\frac{N_{u}^{(i)}}{2}-1\right)}\right]^{T}
$$

and first row

$$
\left[u_{\frac{N_{u}^{(i)}}{2}}^{(i)}, \cdots, u_{0}^{(i)}, \mathbf{0}_{1 \times\left(L^{(i)}-\frac{N_{u}^{(i)}}{2}-1\right)}\right]^{T} .
$$

It is a matrix in which the nonzero entries are only on main diagonal and the first $\frac{N_{u}^{(i)}}{2}$ diagonals above and below.

The backward spreading matrix $\mathbf{U}^{(i: l)}$ is a strictly lower triangular matrix with its $(r, c)$-th element being defined as

$$
U_{r, c}^{(i: l)}= \begin{cases}u_{\frac{N_{u}^{(i)}}{2}-1-L^{(i)}+r-c}^{(i)}, & r \geq c+L^{(i)}-\frac{N_{u}^{(i)}}{2}+1 \\ 0, & r<c+L^{(i)}-\frac{N_{u}^{(i)}}{2}+1 \\ & \left(0 \leq r, c \leq L^{(i)}-1\right) .\end{cases}
$$

3) Multiplexing of mixed numerologies: Signals from all numerologies are added prior to RF transmission. Assume that multiplexing different numerologies is performed within a subframe. As shown in Fig. 3, numerologies have different symbol durations. OFDM symbols of a numerology with smaller subcarrier spacing have a longer symbol duration, which makes OFDM symbols overlap in a more complicated way compared to a single numerology scenario. Taking the symbol duration of the $i$-th numerology as a reference, we can divide the set of numerologies $\mathcal{S}_{\text {num }}$ into two subsets $\mathcal{S}_{\text {num }}^{i-}$ and $\mathcal{S}_{\text {num }}^{i+}$ with a smaller and greater subcarrier spacing than the $i$-th numerology, respectively. They are defined as

$$
\begin{aligned}
& \mathcal{S}_{\text {num }}^{(i-)}=\left\{j: \mu^{(j)}<\mu^{(i)} ; i, j \in \mathcal{S}_{\text {num }}\right\}, \\
& \mathcal{S}_{\text {num }}^{(i+)}=\left\{j: \mu^{(j)}>\mu^{(i)} ; i, j \in \mathcal{S}_{\text {num }}\right\} .
\end{aligned}
$$




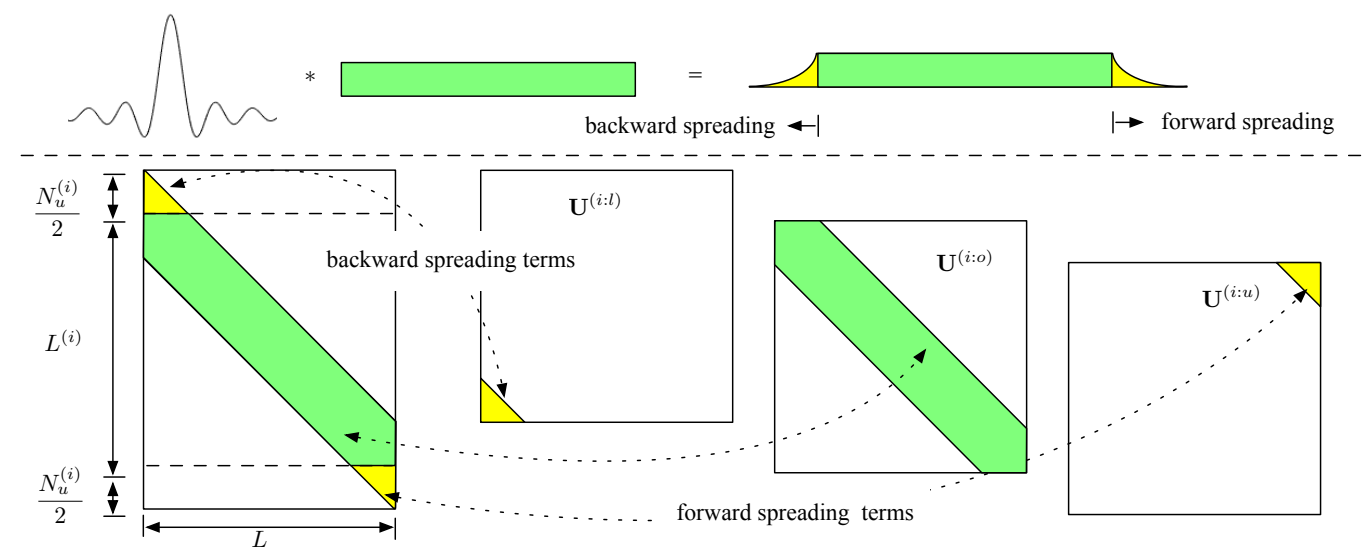

Fig. 2. Matrix shapes of filter forward/backward spreading

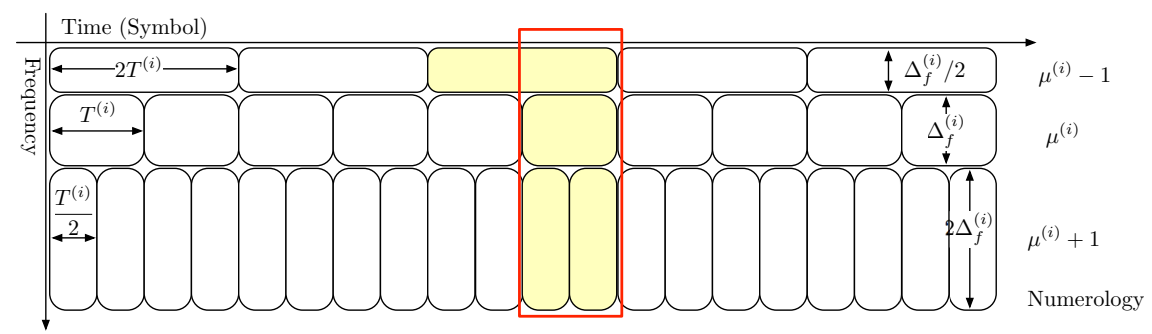

Fig. 3. An example of symbol overlap among different numerologies.

Assume perfect synchronization for all numerologies, we have the following propositions for multiplexing two numerologies (proof can be found in Appendix A):

Proposition 1. The symbol duration of the $j$-th numerology $\left(j \in \mathcal{S}_{\text {num }}^{(i-)}\right)$ is $\frac{\nu^{(i)}}{\nu^{(j)}}$ times long as that of the $i$-th numerology. If we divide each OFDM symbol of the $j$-th numerology into $\frac{\nu^{(i)}}{\nu^{(j)}}$ symbol parts $(S P S)$ of the same length, then the $\left(k \bmod \frac{\nu^{(i)}}{\nu^{(j)}}\right)$ th SP of the $\left(\left\lceil k \frac{\nu^{(j)}}{\nu^{(i)}}\right\rceil\right)$-th OFDM symbol, denoted as $\mathbf{s}_{k}^{(i \leftarrow j)}$, exactly overlaps with the $k$-th OFDM symbol of the $i$-th numerology. The SP $\mathbf{s}_{k}^{(i \leftarrow j)}$ can be obtained by slicing the corresponding part from the $\left(k \bmod \frac{\nu^{(i)}}{\nu^{(j)}}\right)$-th OFDM symbol of the $j$-th numerology, $\mathbf{s}_{\left\lceil k \frac{\nu^{(j)}}{\nu^{(i)}}\right\rceil}^{(j)}$, as

$$
\mathbf{s}_{k}^{(i \leftarrow j)}=\mathbf{C}_{k}^{(i \leftarrow j)} \mathbf{s}_{\left\lceil k \frac{\nu^{(j)}}{\nu^{(i)}}\right\rceil}^{(j)}, \quad j \in \mathcal{S}_{\text {num }}^{(i-)},
$$

where $\mathbf{C}_{k}^{(i \leftarrow j)}$ is an $L^{(i)} \times L^{(j)}$ dimensional slicing matrix defined as

$$
\mathbf{C}_{k}^{(i \leftarrow j)}=\left[\mathbf{0}_{\text {left }}, \mathbf{I}_{L^{(i)}}, \mathbf{0}_{\text {right }}\right],
$$

where $\mathbf{0}_{\text {left }}$ is a $L^{(i)} \times\left(k \bmod \frac{\nu^{(i)}}{\nu^{(j)}}\right) L^{(i)}$ zero matrix, $\mathbf{0}_{\text {right }}$ is $a L^{(i)} \times\left(L^{(j)}-\left(k \bmod \frac{\nu^{(i)}}{\nu^{(j)}}\right)+1\right) L^{(i)}$ zero matrix.

Proposition 2. The symbol duration of the $i$-th numerology is $\frac{\nu^{(j)}}{\nu^{(i)}}$ times long as that of the $j$-th numerology $\left(j \in \mathcal{S}_{\text {num }}^{(i+)}\right)$. The $k$-th OFDM symbol of the $i$-th numerology overlaps with $\frac{\nu^{(j)}}{\nu^{(i)}}$ symbols of the $j$-th numerology in the range of $\frac{k \nu^{(j)}}{\nu^{(i)}}, \frac{k \nu^{(j)}}{\nu^{(i)}}+$ $1, \cdots, \frac{(k+1) \nu^{(j)}}{\nu^{(i)}}-1$. Grouping these symbols into an $L^{(i)} \times 1$ vector $\mathbf{s}_{k}^{(i \stackrel{\nu}{\leftarrow} j)}$ yields

$$
\begin{aligned}
& \mathbf{s}_{k}^{(i \leftarrow j)}=\left[\left(\mathbf{s}_{\frac{k \nu}{\nu^{(i)}}}^{(j)}\right)^{T},\left(\mathbf{s}_{\frac{k \nu}{\nu^{(i)}}+1}^{(j)}\right)^{T}, \cdots,\right. \\
& \left.\left(\mathbf{s}_{\frac{(k+1) \nu(j)}{\nu^{(i)}}-1}^{(j)}\right)^{T}\right]^{T}, \quad j \in \mathcal{S}_{\text {num }}^{(i+)} .
\end{aligned}
$$

By multiplexing signals of all numerologies, the mixed signal with respect to the $k$-th OFDM symbol of the $i$-th numerology can be written as

$$
\mathbf{s}_{k}^{<i>}=\mathbf{s}_{k}^{(i)}+\tilde{\mathbf{s}}_{k}^{(i)}
$$

where

$$
\tilde{\mathbf{s}}_{k}^{(i)}=\sum_{j \in \mathcal{S} \backslash\{i\}} \mathbf{s}_{k}^{(i \leftarrow j)}
$$

is the multiplexing signal from numerologies other than the $i$-th numerology.

\section{B. Passing the Signal Through the Channel}

An $\left(N_{\mathrm{ch}}^{(i)}+1\right)$-tap channel between the transmitter and the receiver of the $i$-th numerology is assumed to have an impulse 
response

$$
\mathbf{h}^{(i)}=\left[h_{0}^{(i)}, h_{1}^{(i)}, \ldots, h_{N_{c h}^{(i)}}^{(i)}\right]^{T} .
$$

After passing through the above channel followed by adding an additive white Gaussian noise (AWGN), the $L^{(i)}$ received samples corresponding to the $k$-th OFDM symbol of the $i$-th numerology can be written as

$$
\mathbf{r}_{k}^{<i>}=\mathbf{H}^{(i: u)} \mathbf{s}_{k-1}^{<i>}+\mathbf{H}^{(i: o)} \mathbf{s}_{k}^{<i>}+\mathbf{w}_{k}^{(i)},
$$

where $\mathbf{H}^{(i: o)}$ is a Toeplitz matrix with $\left[\left(\mathbf{h}^{(i)}\right)^{T}, \mathbf{0}_{1 \times\left(L-N_{\mathrm{ch}}-1\right)}\right]^{T} \in \mathbb{C}^{L^{(i)} \times 1}$ being its first column and $\left[h_{0}^{(i)}, \mathbf{0}_{1 \times(L-1)}\right]^{T} \in \mathbb{C}^{L^{(i)} \times 1}$ being its first row. The channel spreading matrix $\mathbf{H}^{(i: u)}$ is a strictly upper triangular matrix with its $(r, c)$-th element defined as

$$
\begin{array}{r}
H_{r, c}^{(i: u)}= \begin{cases}h_{L^{(i)}+r-c}^{(i)}, & c \geq r+L^{(i)}-N_{\mathrm{ch}}^{(i)} \\
0, & \text { otherwise }\end{cases} \\
\quad\left(0 \leq r, c \leq L^{(i)}-1\right) .
\end{array}
$$

and $\mathbf{w}_{k}^{(i)}$ is the complex Gaussian noise vector with each element having a zero mean and variance $\sigma_{n}^{2}$.

By replacing $\mathbf{s}_{k}^{<i>}$ in (17) with its expression in (14), we can decompose the vector $\mathbf{r}_{k}^{<i>}$ as

$$
\mathbf{r}_{k}^{<i>}=\mathbf{r}_{k}^{(i)}+\tilde{\mathbf{r}}_{k}^{(i)}+\mathbf{w}_{k}^{(i)}
$$

where

$$
\begin{aligned}
& r_{k}^{(i)}=\mathbf{H}^{(i: u)} \mathbf{s}_{k-1}^{(i)}+\mathbf{H}^{(i: o)} \mathbf{s}_{k}^{(i)}, \\
& \tilde{r}_{k}^{(i)}=\mathbf{H}^{(i: u)} \tilde{\mathbf{s}}_{k-1}^{(i)}+\mathbf{H}^{(i: o)} \tilde{\mathbf{s}}_{k}^{(i)},
\end{aligned}
$$

refer to the contributions to $\mathbf{r}_{k}^{<i>}$ from the signal of the $i$-th numerology and the others, respectively.

\section{Receiver Baseband Processing}

1) Filtering at the receiver: The primary purpose of the filtering at the receiver is to reject the signal contribution from other numerologies. As mentioned earlier that any filter processing at the transmitter is not known at the receiver in the transparent design, we define an independent receiver filter of length $N_{v}^{(i)}$ as

$$
\mathbf{v}^{(i)}=\left[v_{0}^{(i)}, v_{1}^{(i)}, \cdots, v_{N_{v}^{(i)}}^{(i)}\right]^{T} .
$$

When no filtering is performed at the receiver, we have $\mathbf{v}^{(i)}=$ [1].

The $L^{(i)}$ samples corresponding to the $k$-th OFDM symbol passing through the receiver filter are grouped into a vector $\mathbf{z}_{k}^{<i>}$, obtaining

$$
\mathbf{z}_{k}^{<i>}=\mathbf{V}^{(i: u)} \mathbf{r}_{k-1}^{<i>}+\mathbf{V}^{(i: o)} \mathbf{r}_{k}^{<i>}+\mathbf{V}^{(i: l)} \mathbf{r}_{k+1}^{<i>},
$$

where matrices $\mathbf{V}$ take the same form as matrices $\mathbf{U}$, and the definition of $\mathbf{V}^{(i, u)}$ and $\mathbf{V}^{(i, l)}$ can be found in Eq. (8) and Eq. (9), respectively, with the notations ' $u / U$ ' being replaced by
'v/V'. Similar to the matrix $\mathbf{U}^{(i: o)}$, the toeplitz matrix $\mathbf{V}^{(i: o)}$ is specified by its first column

$$
\left[v_{\frac{N_{v}^{(i)}}{2}}^{(i)}, \cdots, v_{N_{v}^{(i)}}^{(i)}, \mathbf{0}_{1 \times\left(L^{(i)}-\frac{N_{v}^{(i)}}{2}-1\right)}\right]^{T}
$$

and first row

$$
\left[v_{\frac{N_{v}^{(i)}}{2}}^{(i)}, \cdots, v_{0}^{(i)}, \mathbf{0}_{1 \times\left(L^{(i)}-\frac{N_{v}^{(i)}}{2}-1\right)}\right]^{T} .
$$

An ideal low-pass filter rejects all signal energy above a designated cut-off frequency. However, in practice, this is impossible to achieve as the required impulse response would be infinitely long. Practical finite-length filters inevitably result in residual signals above the cut-off frequency. Thus, $\mathbf{z}_{k}^{(i)} \mathrm{com}$ prises signal components not only from the $i$-th numerology, but also from other numerologies. Denote $\mathbf{z}_{k}$ as the sum of three terms, i.e.,

$$
\mathbf{z}_{k}^{<i>}=\mathbf{z}_{k}^{(i)}+\tilde{\mathbf{z}}_{k}^{(i)}+\tilde{\mathbf{w}}_{k}^{(i)}
$$

where

$$
\begin{gathered}
\mathbf{z}_{k}^{(i)}=\mathbf{V}^{(i: u)} \mathbf{r}_{k-1}^{(i)}+\mathbf{V}^{(i: o)} \mathbf{r}_{k}^{(i)}+\mathbf{V}^{(i: l)} \mathbf{r}_{k+1}^{(i)} \\
\tilde{\mathbf{z}}_{k}^{(i)}=\mathbf{V}^{(i: u)} \tilde{\mathbf{r}}_{k-1}^{(i)}+\mathbf{V}^{(i: o)} \tilde{\mathbf{r}}_{k}^{(i)}+\mathbf{V}^{(i: l)} \tilde{\mathbf{r}}_{k+1}^{(i)}
\end{gathered}
$$

correspond to the signal components from the $i$-th numerology and the residual interference signal to the $i$-th numerology, respectively. Their expression prior to any filtering operation are derived in Appendix B and Appendix C. The filtered noise $\tilde{\mathbf{w}}_{k}^{(i)}$ can be expressed as

$$
\tilde{\mathbf{w}}_{k}^{(i)}=\mathbf{V}^{(i: u)} \mathbf{w}_{k-1}^{(i)}+\mathbf{V}^{(i: o)} \mathbf{w}_{k}^{(i)}+\mathbf{V}^{(i: l)} \mathbf{w}_{k+1}^{(i)} .
$$

2) CR removal and DFT: After removing the $C R$ and performing the FFT operation, the received symbol vector corresponding to the $k$-th OFDM of the $i$-th numerology can be obtained as

$$
\mathbf{y}_{k}^{<i>}=\left(\mathbf{F}^{(i)}\right)^{H} \mathbf{R}_{\mathrm{cr}}^{(i)} \mathbf{z}_{k}^{<i>},
$$

where the CR removal matrix is formed as

$$
\mathbf{R}_{\mathrm{cr}}^{(i)}=\left[\mathbf{0}_{N^{(i)} \times N_{\mathrm{cp}}^{(i)}}, \mathbf{I}_{N^{(i)}}, \mathbf{0}_{N^{(i)} \times N_{\mathrm{cs}}^{(i)}}\right] \in \mathbb{B}^{N^{(i)} \times L^{(i)}} .
$$

After some algebraic manipulations based on equations from (4) to (28), the received signal $\mathbf{y}_{k}^{<i>}$ can be decomposed as

$$
\mathbf{y}_{k}^{<i>}=\underbrace{\mathbf{y}_{k, \text { des }}^{(i)}}_{\text {desired signal }}+\underbrace{\mathbf{y}_{k, \text { intra }}^{(i)}}_{\text {IntraNI signal }}+\underbrace{\mathbf{y}_{k, \text { inter }}^{(i)}}_{\text {InterNI signal }}+\underbrace{\hat{\mathbf{w}}_{k}^{(i)}}_{\text {noise }} .
$$

The desired signal can be expressed as

$$
\mathbf{y}_{k, \mathrm{des}}^{(i)}=\boldsymbol{\Psi}_{\mathrm{des}}^{(i)} \mathbf{d}_{k}^{(i)}
$$

where the $N_{c}^{(i)} \times N_{c}^{(i)}$ dimensional matrix $\boldsymbol{\Psi}_{\text {des }}^{(i)}$, which is the equivalent channel matrix to the desired signal, can be expressed as

$$
\boldsymbol{\Psi}_{\mathrm{des}}^{(i)}=\rho_{\mathrm{cr}}^{(i)}\left(\mathbf{F}^{(i)}\right)^{H} \mathbf{R}_{\mathrm{cr}}^{(i)} \boldsymbol{\Theta}^{(i)} \mathbf{T}_{\mathrm{cr}}^{(i)} \mathbf{F}^{(i)},
$$


with

$$
\begin{aligned}
\boldsymbol{\Theta}^{(i)}= & \mathbf{V}^{(i: u)} \mathbf{H}^{(i: o)} \mathbf{U}^{(i: l)}+\mathbf{V}^{(i: o)} \mathbf{H}^{(i: u)} \mathbf{U}^{(i: l)} \\
& +\mathbf{V}^{(i: o)} \mathbf{H}^{(i: o)} \mathbf{U}^{(i: o)}+\mathbf{V}^{(i: l)} \mathbf{H}^{(i: o)} \mathbf{U}^{(i: u)} .
\end{aligned}
$$

The derivation can be found in Appendix B. The interference components and the noise in (29) will be discussed in Section III and Section IV.

3) Equalization and detection: The well-channelized signal (29) can be equalized using the conventional equalization methods with a trade-off between complexity and performance. The simplest method is the one-tap equalization using the diagonal matrix $\boldsymbol{\Psi}_{\text {des }}$, i.e.,

$$
\hat{\mathbf{d}}_{k}^{(i)}=\boldsymbol{\Psi}_{\mathrm{des}}^{-1} \mathbf{y}_{k}^{(i)},
$$

which can be performed independently on each subcarrier.

\section{ANALYSIS OF NOISE AND INTRANI INTERFERENCE IN F-OFDM SYSTEMS}

In this section, we briefly discuss the noise and the IntraNI which only occurs within a numerology in f-OFDM systems before proceeding to our main discussion on the InterNI in the next section.

\section{A. Noise Distribution in f-OFDM Systems}

According to (27), we can see that the noise is not identically distributed across subcarriers. The $N_{c}^{(i)} \times 1$ complex noise vector added on the received data symbols of the $k$-th f-OFDM symbol can be reformulated as

$$
\begin{aligned}
\hat{\mathbf{w}}_{k}^{(i)}=\left(\mathbf{F}^{(i)}\right)^{H} \mathbf{R}_{\mathrm{cr}}^{(i)}\left(\mathbf{V}^{(i: u)} \mathbf{w}_{k-1}\right. \\
\left.+\mathbf{V}^{(i: o)} \mathbf{w}_{k}+\mathbf{V}^{(i: l)} \mathbf{w}_{k+1}\right) \\
=\left(\mathbf{F}^{(i)}\right)^{H} \mathbf{R}_{\mathrm{cr}}^{(i)} \mathbf{V}^{(i:[u, m, l])} \mathbf{w}_{[k-1, k, k+1]}^{(i)},
\end{aligned}
$$

where the $L^{(i)} \times 3 L^{(i)}$ matrix

$$
\mathbf{V}^{(i:[u, m, l])}=\left[\mathbf{V}^{(i: u)}, \mathbf{V}^{(i: o)}, \mathbf{V}^{(i: l)}\right]
$$

The $3 L^{(i)} \times 1$ vector

$$
\mathbf{w}_{[k-1, k, k+1]}^{(i)}=\left[\left(\mathbf{w}_{k-1}^{(i)}\right)^{T},\left(\mathbf{w}_{k}^{(i)}\right)^{T},\left(\mathbf{w}_{k+1}^{(i)}\right)^{T}\right]^{T}
$$

is a complex Gaussian random vector with

$$
\mathbf{w}_{k}^{(i, c)} \sim \mathcal{C N}\left(\mathbf{0}_{3 L^{(i)} \times 1}, \sigma_{n}^{2} \mathbf{I}_{3 L^{(i)}}\right) .
$$

The matrix multiplication, $\left(\mathbf{F}^{(i)}\right)^{H} \mathbf{R}_{\mathrm{cr}}^{(i)} \mathbf{V}^{(i:[u, m, l])}$, results in a complex matrix. According to [30], $\hat{\mathbf{w}}_{k}^{(i)}$ is also Gaussian, with covariance matrix $\mathbf{C}_{w}^{(i)}$, i.e., $\mathcal{C N}\left(\mathbf{0}, \mathbf{C}_{w}^{(i)}\right)$, where the covariance matrix $\mathbf{C}_{w}^{(i)} \in \mathbb{C}^{N_{c}^{(i)} \times N_{c}^{(i)}}$ can be computed as

$$
\begin{aligned}
\mathbf{C}_{w}^{(i)}= & \mathcal{E}\left\{\hat{\mathbf{w}}_{k}^{(i)}\left(\hat{\mathbf{w}}_{k}^{(i)}\right)^{H}\right\} \\
= & \left(\mathbf{F}^{(i)}\right)^{H} \mathbf{R}_{\mathrm{cr}}^{(i)} \mathbf{V}^{(i:[u, m, l])} \\
& \left(\mathbf{V}^{(i:[u, m, l])}\right)^{H}\left(\mathbf{R}_{\mathrm{cr}}^{(i)}\right)^{H} \mathbf{F}^{(i)} \sigma_{n}^{2} .
\end{aligned}
$$

\section{B. Channel diagonization and IntraNI-free systems}

Additional filtering on top of the OFDM waveform spreads symbol bi-directionally as illustrated in Fig. 2. The intraNI occurs when the spreading exceeds the coverage of CR. Extended $\mathrm{CR}$ can be introduced to combat the interference. When the extended CR is sufficiently long to cover all the spreading of the filters, the IntraNI is eliminated $\left(\mathbf{y}_{k \text {,intra }}^{(i)}=0\right)$, and IntraNI-free transmission is achieved. The linear convolution, $\mathbf{v}^{(i)} * \mathbf{h}^{(i)} * \mathbf{u}^{(i)} * \mathbf{x}^{(i)}$, can then be converted into a circular convolution, $\mathbf{v}^{(i)} \circledast \mathbf{h}^{(i)} \circledast \mathbf{u}^{(i)} \circledast \mathbf{x}^{(i)}$. Thus, the desired signal in (29) can be diagonalized and expressed as

$$
\mathbf{y}_{k, \mathrm{des}}^{(i)}=\boldsymbol{\Lambda}^{(i)} \mathbf{d}_{k}^{(i)}
$$

where the $N_{c}^{(i)} \times N_{c}^{(i)}$ dimensional diagonal channel matrix $\mathbf{\Lambda}^{(i)}=\operatorname{diag}(\ddot{\mathbf{v}}) \operatorname{diag}(\ddot{\mathbf{h}}) \operatorname{diag}(\ddot{\mathbf{u}})$ with $\ddot{\mathbf{t}}, \mathbf{t} \in\{\mathbf{u}, \mathbf{v}, \mathbf{h}\}$, refers to the DFT of $\mathbf{t}$ on the corresponding subcarriers of the $i$-th BWP. The power of desired data symbols can be computed as

$$
\left|\mathbf{y}_{k, \mathrm{des}}^{(i)}\right|^{2}=\operatorname{diag}\left(\left(\boldsymbol{\Lambda}^{(i)}\right)^{H} \boldsymbol{\Lambda}^{(i)}\right)\left(\sigma_{s}^{(i)}\right)^{2} \text {. }
$$

The implementation of CR comes with a bandwidth and power penalty. For an f-OFDM system with a stringent frequency localization requirement, the filter length is normally chosen as up to half of the symbol duration, making it unaffordable to satisfy the interference-free condition. However, most of the power of a sinc filter lies within its middle part, and over 90 percent within its main lobe [31]. Therefore, a nearly IntraNI-free system can be achieved if the CR covers the main lobe and first few sidelobes of a filter, and the received data symbol can be approximated by (36) with trivial interference sufficiently small to be ignored [15].

\section{INTER-NUMEROLOGY INTERFERENCE ANALYSIS}

InterNI to a numerology refers to the signal components contributed by other numerologies. When a multiplexing signal is generated from several different numerologies, the orthogonality between subcarriers no longer holds. Subcarriers from one numerology interfere with those from the others. When mixed numerologies are implemented in OFDM systems, the energy leaked from one numerology to the other, a.k.a, out of band (OOB) emission, is high, due to the spreading characteristic of sinc filters. However, in f-OFDM systems, each numerology is accommodated in a subband with a better-localized filter, thus the level of interference between different numerologies is reduced. This motivates us to compare and quantify the interference level in f-OFDM and OFDM systems. We derive the analytical expression of the interNI signal in multi-numerology systems in the sequel.

\section{A. The expression of the InterNI signal}

The contribution from the $j$-th numerology to the received signal of the $i$-th numerology in the $k$-th OFDM window at 
the receiver, denoted as $\mathbf{z}_{k}^{(i \leftarrow j)}$, can be approximated as

$$
\begin{aligned}
& \mathbf{z}_{k}^{(i \leftarrow j)} \approx \\
& \begin{cases}\mathbf{V}^{(i: o)} \mathbf{H}^{(i: o)} \mathbf{C}_{k}^{(i \leftarrow j)} \mathbf{U}^{(j: o)} \mathbf{x}_{k}^{(i \leftarrow j)}, & j \in \mathcal{S}_{\text {num }}^{(i-)} \\
\mathbf{V}^{(i: o)} \mathbf{H}^{(i: o)} \operatorname{blkdiag}\left(\mathbf{U}^{(j: o)}, \frac{\nu^{(j)}}{\nu^{(i)}}\right) \mathbf{x}_{k}^{(i \leftarrow j)}, & j \in \mathcal{S}_{\text {num }}^{(i+)},\end{cases}
\end{aligned}
$$

where, for $j \in \mathcal{S}_{\text {num }}^{(i-)}$,

$$
\mathbf{x}_{k}^{(i \leftarrow j)}=\mathbf{x}_{\left\lceil k \frac{\nu(j)}{\nu(i)}\right\rceil}^{(j)}
$$

for $j \in \mathcal{S}_{\text {num }}^{(i+)}$,

$$
\mathbf{x}_{k}^{(i \leftarrow j)}=\left[\left(\mathbf{x}_{\frac{k \nu(j)}{\nu^{(i)}}}^{(j)}\right)^{T},\left(\mathbf{x}_{\frac{k \nu(j)}{\nu(i)}+1}^{(j)}\right)^{T}, \cdots,\left(\mathbf{x}_{\frac{(k+1) \nu}{\nu^{(i)}}-1}^{(j)}\right)^{T}\right]^{T} .
$$

The detailed derivation can be found in Appendix C.

After removing the CR and performing the FFT operation, the interference symbol vector from the $j$-th numerology to the $i$-th numerology in the frequency domain can be written as

$$
\mathbf{y}_{k, \text { inter }}^{(i \leftarrow j)}=\left(\mathbf{F}^{(i)}\right)^{H} \mathbf{R}_{\mathrm{cr}}^{(i)} \mathbf{z}_{k}^{(i \leftarrow j)}
$$

After conducting some substitutions based on (4), (38), we can express the interNI symbol vector $\mathbf{y}_{k, \text { inter }}^{(i \leftarrow j)}$ in (39) as

$$
\mathbf{y}_{k, \text { inter }}^{(i \leftarrow j)}=\Psi_{k}^{(i \leftarrow j)} \mathbf{d}_{k}^{(i \leftarrow j)} .
$$

where

$$
\boldsymbol{\Psi}_{k}^{(i \leftarrow j)}=\left\{\begin{array}{c}
\rho_{\mathrm{cr}}^{(j)}\left(\mathbf{F}^{(i)}\right)^{H} \mathbf{R}_{\mathrm{cr}}^{(i)} \mathbf{V}^{(i: o)} \mathbf{H}^{(i: o)} \\
\mathbf{C}_{k}^{(i \leftarrow j)} \mathbf{U}^{(j: o)} \mathbf{T}_{\mathrm{cr}}^{(j)} \mathbf{F}^{(j)}, j \in \mathcal{S}_{\mathrm{num}}^{(i-)} \\
\rho_{\mathrm{cr}}^{(j)}\left(\mathbf{F}^{(i)}\right)^{H} \mathbf{R}_{\mathrm{cr}}^{(i)} \mathbf{V}^{(i: o)} \mathbf{H}^{(i: o)} \\
\quad \operatorname{blkdiag}\left(\mathbf{U}^{(j: o)} \mathbf{T}_{\mathrm{cr}}^{(j)} \mathbf{F}^{(j)}, \frac{\nu^{(j)}}{\nu^{(i)}}\right), j \in \mathcal{S}_{\text {num }}^{(i+)}
\end{array}\right.
$$

and

$$
\mathbf{d}_{k}^{(i \leftarrow j)}=\left\{\begin{array}{cl}
\mathbf{d}_{\left\lceil k \frac{\nu(j)}{\nu(i)}\right\rceil}^{(j)}, & j \in \mathcal{S}_{\text {num }}^{(i-)} \\
{\left[\left(\mathbf{d}_{\frac{k \nu(j)}{\nu^{(i)}}}^{(j)}\right)^{T},\left(\mathbf{d}_{\frac{k \nu(j)}{\nu^{(i)}}+1}^{(j)}\right)^{T},\right.} & \\
\left.\cdots,\left(\mathbf{d}_{\left.\frac{(k+1) \nu}{\nu^{(i)}}-1\right)}^{(j)}\right)^{T}\right]^{T}, & j \in \mathcal{S}_{\text {num }}^{(i+)}
\end{array}\right.
$$

The matrix $\Psi_{k}^{(i \leftarrow j)} \in \mathbb{C}^{N_{c}^{(i)} \times\left(\left\lceil\frac{\nu^{(j)}}{\nu^{(i)}}\right\rceil M^{(j)}\right)}$ can be interpreted as the equivalent channel which transforms data symbols from the $j$-th numerology into the InterNI to the $i$-th numerology in the $k$-th OFDM symbol. The vector $\mathbf{d}_{k}^{(i \leftarrow j)} \in \mathbb{C}^{\left(\left\lceil\frac{\nu^{(j)}}{\nu^{(i)}}\right\rceil M^{(j)}\right) \times 1}$ comprises those data symbols from the $j$-th numerology which generate interference to the symbols in the $k$-th OFDM window of the $i$-th numerology.

Adding the interference components from all the other numerologies yields the expression of total interNI to the $i$-th numerology as

$$
\mathbf{y}_{k, \text { inter }}^{(i)}=\sum_{j \in \mathcal{S}_{\text {num }} \backslash\{i\}} \Psi_{k}^{(i \leftarrow j)} \mathbf{d}_{k}^{(i \leftarrow j)} .
$$

\section{B. The analytical expression of the InterNI power}

The instantaneous power of the InterNI signal contributed from the $j$-th numerology to all the subcarriers in the $k$-th OFDM symbol of the $i$-th numerology can be grouped into an $N_{c}^{(i)}$ dimensional vector as

$$
\begin{aligned}
& \left|\mathbf{y}_{k, \text { InterNI }}^{(i \leftarrow j)}\right|^{2}= \\
& \operatorname{diag}\left(\boldsymbol{\Psi}_{k}^{(i \leftarrow j)} \mathbf{d}_{k}^{(i \leftarrow j)}\left(\mathbf{d}_{k}^{(i \leftarrow j)}\right)^{H}\left(\mathbf{\Psi}_{k}^{(i \leftarrow j)}\right)^{H}\right) .
\end{aligned}
$$

The average level of distortion caused by the interfering numerologies can be expressed as a function of these parameters as

$$
\begin{array}{r}
\left|\overline{\mathbf{y}}_{k, \text { InterNI }}^{(i \leftarrow j)}\left(N_{c}^{(a)}, G^{(a)}, \mathbf{u}^{(j)}, \mathbf{v}^{(i)}, \mathbf{h}^{(i)},\left(\sigma_{s}^{(j)}\right)^{2}\right)\right|^{2} \\
=\mathcal{E}\left\{\operatorname{diag}\left(\mathbf{\Psi}_{k}^{(i \leftarrow j)}\left(\mathbf{\Psi}_{k}^{(i \leftarrow j)}\right)^{H}\right)\right\}\left(\sigma_{s}^{(j)}\right)^{2}, \\
a \in\{i, j\} .
\end{array}
$$

The identical power spectrum distribution (PWD) property of fading channel, i.e. $\mathcal{E}\left\{\ddot{\mathbf{h}}^{H}\right\}=\mathbf{I}$, indicates that the interference power in (45) can be rewritten as

$$
\begin{array}{r}
\left|\overline{\mathbf{y}}_{k, \text { InterNI }}^{(i \leftarrow j)}\left(N_{c}^{(a)}, G^{(a)}, \mathbf{u}^{(j)}, \mathbf{v}^{(i)}, \mathbf{h}^{(i)},\left(\sigma_{s}^{(j)}\right)^{2}\right)\right|^{2} \\
=\operatorname{diag}\left(\tilde{\mathbf{\Psi}}_{k}^{(i \leftarrow j)}\left(\tilde{\mathbf{\Psi}}_{k}^{(i \leftarrow j)}\right)^{H}\right)\left(\sigma_{s}^{(j)}\right)^{2}, \\
a \in\{i, j\},
\end{array}
$$

where, for $j \in \mathcal{S}_{\text {num }}^{(i-)}$,

$$
\tilde{\boldsymbol{\Psi}}_{k}^{(i \leftarrow j)}=\rho_{\mathrm{cr}}^{(j)}\left(\mathbf{F}^{(i)}\right)^{H} \mathbf{R}_{\mathrm{cr}}^{(i)} \mathbf{V}^{(i: o)} \mathbf{C}_{k}^{(i \leftarrow j)} \mathbf{U}^{(j: o)} \mathbf{T}_{\mathrm{cr}}^{(j)} \mathbf{F}^{(j)},
$$

for $j \in \mathcal{S}_{\text {num }}^{(i+)}$,

$$
\begin{aligned}
\tilde{\mathbf{\Psi}}_{k}^{(i \leftarrow j)}=\rho_{\mathrm{cr}}^{(j)}\left(\mathbf{F}^{(i)}\right)^{H} & \mathbf{R}_{\mathrm{cr}}^{(i)} \mathbf{V}^{(i: o)} \\
& \text { blkdiag }\left(\mathbf{U}^{(j: o)} \mathbf{T}_{\mathrm{cr}}^{(j)} \mathbf{F}^{(j)}, \frac{\nu^{(j)}}{\nu^{(i)}}\right) .
\end{aligned}
$$

The above expression for the InterNI and the expression of $\Psi_{k}^{(i \leftarrow j)}$ in (41) implies that, although not in an explicit manner, some parameters may influence the InterNI level. We provide some qualitative explanations of the influences from these parameters in the following analysis, and the quantitative evaluation will be presented in the Section VI. Firstly, the matrices $\mathbf{V}^{(i: o)}$ and $\mathbf{U}^{(j: o)}$ (correspond to the receiver filtering in the $i$ th numerology and transmitter filtering in the $j$-th numerology, respectively) indicate that filter spectrum localization property, dependent on filter length and roll-off parameters, may affect the InterNI level. Secondly, parameters including subcarrier spacing, guard band and BWP width, define the matrices $\mathbf{F}^{(i)}$ and $\mathbf{F}^{(j)}$. These parameters may also impact the signal distortion level, as they either indicate the root cause of nonorthogonality or measure the isolation distance between the two numerologies in the frequency domain. Finally, the power allocated to the interference numerology $\left(\sigma_{s}^{(j)}\right)^{2}$ also has an effect on the interference level to the numerology of interest. 


\section{The metrics of the signal distortion due to the InterNI}

To quantify the signal distortion due to the in-band unwanted emissions, i.e., the interference between different numerologies/BWPs, we adopt the metric: signal to InterNI ratio (SIR). The SIR is defined as the ratio of the observable reference power on the $k$-th OFDM symbol of $\mathrm{Rx} \mathrm{BWP}^{(i)}$ to the power leaking from an interfering $\mathrm{Tx} \mathrm{BWP}^{(j)}$, i.e.,

$$
\operatorname{SIR}_{k, \text { InterNI }}^{(i \leftarrow j)}=10 \log _{10}\left(\frac{\left|\mathbf{y}_{k, \text { des }}^{(i)}\right|^{2}}{\left|\mathbf{y}_{k, \text { InterNI }}^{(i \leftarrow j)}\right|^{2}}\right),
$$

where the reference power on the active subcarriers of the $k$-th OFDM symbol on $\mathrm{Rx} \mathrm{BWP}^{(i)}$ is obtained as

$$
\left|\mathbf{y}_{k, \operatorname{des}}^{(i)}\right|^{2}=\operatorname{diag}\left(\boldsymbol{\Psi}_{\mathrm{des}}^{(i)}\left(\boldsymbol{\Psi}_{\mathrm{des}}^{(i)}\right)^{H}\right)\left(\sigma_{s}^{(i)}\right)^{2}
$$

and the power leaking from active subcarriers on $\operatorname{Tx} \mathrm{BWP}^{(j)}$ to the active subcarriers of the $k$-th OFDM symbol on $\mathrm{Rx}$ $\mathrm{BWP}^{(i)}$ is defined in (46).

It is worth pointing out that the interNI level for multinumerology OFDM systems can be obtained by replacing filtering matrices with identity matrices. Therefore, our analytical work is valid for both OFDM and f-OFDM.

\section{Further discussion on the interference in multi-numerology f-OFDM systems}

Although the main focus of the paper is on the analysis of the InterNI, in practice, a trade-off must be considered between the InterNI and IntraNI. We will investigate this issue in our future work where the mathematical expression of the residual IntraNI (filter spreading not covered by the CR) will be derived by following the same procedure for the InterNI. With the expressions of both the IntraNI and InterNI, filters can be optimized to strike a good balance between the InterNI and IntraNI, while meeting the minimum requirements on signal quality specified in 3GPP [19] (e.g., spectral and temporal masks (SEM), adjacent channel leakage ratio (ACLR), error vector magnitude (EVM)).

When analyzing the signal corruption due to the InterNI, we assume that the deployed CR covers most of the filter energy so that the residual IntraNI is very limited and can be safely ignored. This assumption is proved to be reasonable by the simulations using 3GPP test parameters in [32]. The level of distortion induced by mixed numerologies, analytically expressed as a function of several system parameters in (46), can be exploited to analyze and evaluate these parameters, i.e., the minimum guard band, the minimum filter length, etc., to meet a given SIR requirement for each numerology. The expression of instantaneous power of the interNI in (44) facilitates the formulation of spectrum/power efficiency optimization problems for multi-numerology systems. It is impossible to investigate all the applications that our analytical work enables in one paper due to the page limitation. However, in what follows, we demonstrate how the developed analytical framework is utilized to formulate an optimization problem on power allocation in multi-numerology f-OFDM/OFDM systems.

\section{A Case Study: Optimizing Power Allocation in THE PRESENCE OF MiXEd NuMEROLOGIES}

In this section, we provide a case study on optimizing power allocation on the downlink in a multi-numerology system. Without loss of generality, we assume that each numerology has one user and the filters applied to each numerology meet the minimum requirements on SEM/ACLR/EVM specified in 3GPP [19]. We further assume that data and guard subcarriers for each numerology have been allocated. We consider a block fading channel which is assumed to be constant during the transmission of a block and independent from block to block. The length of a block lasts over the duration of several OFDM symbols of the greatest length among all the numerologies. Power is allocated on a block basis in the base station (BS). We define an $M \times N_{c}^{(i)}$ dimensional power allocation vector $\mathbf{p}=\left[\left(\mathbf{p}^{(0)}\right)^{T},\left(\mathbf{p}^{(1)}\right)^{T}, \cdots,\left(\mathbf{p}^{(M-1)}\right)^{T}\right]^{T}$, where the $N_{c}^{(i)}$ dimensional vector $\mathbf{p}^{(i)}=\left[p_{0}^{(i)}, p_{1}^{(i)}, \cdots, p_{N_{c}^{(i)}-1}^{(i)}\right]^{T}$ corresponds to the power allocation to each subcarrier in $\mathrm{BWP}^{(i)}$.

According to (29), we can express the QAM symbol received on the $m$-th subcarrier in the $k$-th OFDM symbol of $\mathrm{BWP}^{(i)}$ as

$$
y_{k, m}^{<i>}=y_{k, m, \text { des }}^{(i)}+y_{k, m, \text { intra }}^{(i)}+y_{k, m, \text { inter }}^{(i)}+\hat{w}_{k, m}^{(i)} .
$$

According to Section III-A, $\hat{w}_{k, m}^{(i)}$ is a complex Gaussian random vector with $\hat{w}_{k, m}^{(i)} \sim \mathcal{C N}\left(0, \mathbf{C}_{w}^{(i)}\right)$. Assume that the $\mathrm{CR}$ is sufficiently long to meet the near IntraNI-free condition discussed in Section III-B. According to (36) and (43), we have the desired signal and the interference signal as

$$
\begin{aligned}
& y_{k, m, \text { intra }}^{(i)} \approx 0, \\
& y_{k, m, \mathrm{des}}^{(i)}=\Lambda_{m, m}^{(i)} \sqrt{p_{m}^{(i)}} \bar{d}_{k, m}^{(i)}, \\
& y_{k, m, \text { inter }}^{(i)}=\sum_{j \in \mathcal{S}_{\text {num } \backslash\{i\}}} \sum_{n=0}^{\left\lceil\frac{\nu^{(j)}}{\nu^{(i)}}\right] M^{(j)}-1} \\
& \Psi_{k, m, n}^{(i \leftarrow j)} \sqrt{p_{n \bmod M^{(j)}}^{(j)}} \bar{d}_{k, n}^{(i \leftarrow j)},
\end{aligned}
$$

where $\bar{d}_{k, m}^{(i)}$ and $\bar{d}_{k, m}^{(i \leftarrow j)}$ are normalized data symbols of $d_{k, m}^{(i)}$ and $d_{k, m}^{(i \leftarrow j)}$, respectively. The signal-to-interference and noise ratio (SINR) on the $m$-th subcarrier in $\mathrm{BWP}^{(i)}$ can be expressed as a function of $\mathbf{p}$, which is written as

$$
\frac{\gamma_{m}^{(i)}(\mathbf{p})=}{\sum_{j \in \mathcal{S}_{\text {num }} \backslash\{i\}} \sum_{n=0}^{\left(i \frac{\nu^{(j)}}{\nu^{(i)}}\right] M^{(j)}-1}\left|\Psi_{k, m, n}^{(i \leftarrow j)}\right|^{2} p_{m}^{(j)} p_{n \bmod M^{(j)}}^{(i)}+\left|\hat{w}_{k, m}^{(i)}\right|^{2}} .
$$

We consider continuous bit-loading and express the achievable bit rate on the $m$-th subcarrier in $\mathrm{BWP}^{(i)}$ as

$$
r_{m}^{(i)}(\mathbf{p})=\Delta f^{(i)} \log \left(1+\gamma_{m}^{(i)}(\mathbf{p})\right)
$$


in the unit of bps (bit per second). The achievable rate for $\mathrm{BWP}^{(i)}$ can then be computed as

$$
\begin{aligned}
R^{(i)}(\mathbf{p}) & =\sum_{m \in \mathcal{M}^{(i)}} r_{m}^{(i)}(\mathbf{p}) \\
& =\sum_{m \in \mathcal{M}^{(i)}} \Delta f^{(i)} \log \left(1+\gamma_{m}^{(i)}(\mathbf{p})\right)
\end{aligned}
$$

bps per channel use.

Problem formulation: Our optimization problem seeks to maximize system sum rate subject to a maximum power constraint. The problem is written as

$$
\begin{aligned}
& \max _{\mathbf{p} \geq 0} \sum_{i \in \mathcal{S}_{\text {num }}} \omega^{(i)} \sum_{m \in \mathcal{M}^{(i)}} \Delta f^{(i)} \log \left(1+\gamma_{m}^{(i)}(\mathbf{p})\right) \\
& \text { s.t. } \sum_{i \in \mathcal{S}_{\text {num }}} \sum_{m \in \mathcal{M}^{(i)}} p_{m}^{(i)} \leq P_{0},
\end{aligned}
$$

Where $P_{0}$ is the maximum transmission power of the system, and each $\omega^{(i)}$ is a tunable non-negative weight that allows a trade-off between the rates allocated to different numerologies. Equivalently, these weights allow the system operators to assign a different QoS level to each numerology.

Replacing $\gamma_{m}^{(i)}(\mathbf{p})$ in (55) with its expression in (52) reveals the objective function as a difference of concave (d.c.) function in $\mathbf{p}$. The problems with d.c. structure have been shown as NP-hard in [33], and a global optimal solution is difficult to achieve. The iterative water falling (IWF) approach [34] achieves an approximate solution by considering this problem as $M$ isolated sub-problems and iterate them until convergence, in which each sub-problem optimizes power allocation $\mathbf{p}^{(i)}$ by treating all other power $\mathbf{p}^{(j \neq i)}$ as fixed noise. We will introduce the approach described in [35] to our power allocation scheme to deal with the d.c. structure and relax the non-convex problem in (55).

The following lower bound is leveraged for relaxing the above non-convex problem

$$
\alpha \log z+\beta \leq \log (1+\gamma) .
$$

It is tight at a chosen $\gamma$ with the constant $\{\alpha, \beta\}$ specified as

$$
\left\{\begin{array}{l}
\alpha=\frac{\gamma}{1+\gamma} \\
\beta=\log (1+\gamma)-\frac{\gamma}{1+\gamma} \log \gamma
\end{array} .\right.
$$

Applying (56) to the optimization problem specified in (55) results in a relaxed problem

$$
\begin{aligned}
& \max _{\mathbf{p} \geq 0} \sum_{i \in \mathcal{S}_{\text {num }}} \sum_{m \in \mathcal{M}^{(i)}} \omega^{(i)} \Delta f^{(i)}\left(\alpha_{m}^{(i)} \log \left(\gamma_{m}^{(i)}(\mathbf{p})\right)+\beta_{m}^{(i)}\right) \\
& \text { s.t. } \sum_{i \in \mathcal{S}_{\text {num }}} \sum_{m \in \mathcal{M}^{(i)}} p_{m}^{(i)} \leq P_{0},
\end{aligned}
$$

where the value of $\alpha_{m}^{(i)}$ and $\beta_{m}^{(i)}$ are fixed for a given p. However, the relaxed problem remains non-convex with respect to $\mathbf{p}$ because SINR function $\gamma_{m}^{(i)}(\mathbf{p})$ is not convex. A

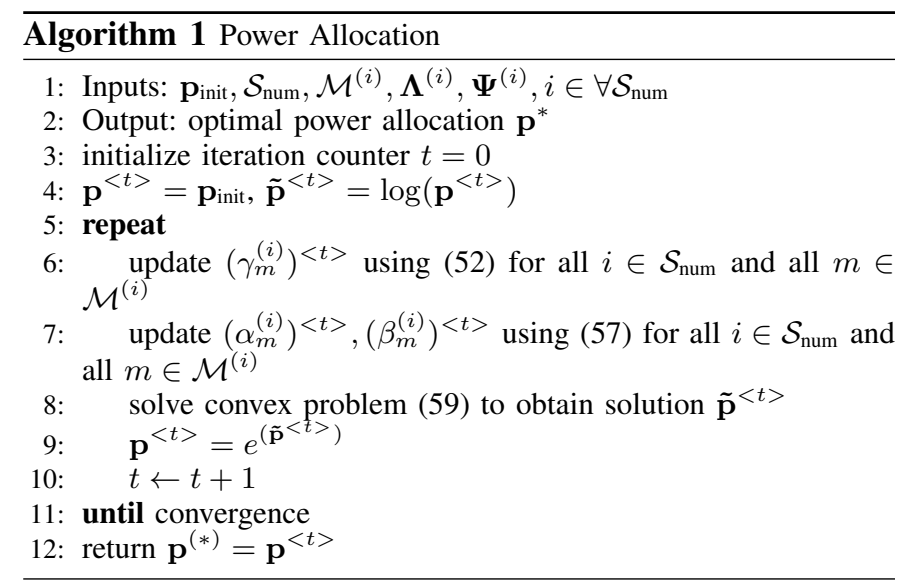

TABLE I

NUMEROLOGY RELATED PARAMETERS

\begin{tabular}{|l||l|l|l|}
\hline Parameters & $\mathrm{BWP}^{\left(a_{1}\right)}$ & $\mathrm{BWP}^{\left(a_{0}\right)}$ & $\mathrm{BWP}^{\left(a_{2}\right)}$ \\
\hline \hline$\mu$ & 2 & 1 & 0 \\
\hline subcarrier spacing (kHz) & 60 & 30 & 15 \\
\hline FFT size & 256 & 512 & 1024 \\
\hline CR length (samples) & 18 & 36 & 72 \\
\hline BWP allocation (kHz) & $0-360$ & $360-540$ & $540-630$ \\
& $(5$ PRBs) & $(5$ PRBs) & $(5$ PRBs $)$ \\
\hline
\end{tabular}

further variable substitution $\mathbf{p}=e^{\tilde{\mathbf{p}}}$ converts the optimization problem into a new function of a variable $\tilde{\mathbf{p}}$ as

$$
\begin{aligned}
& \max _{\tilde{\mathbf{p}} \geq 0} \sum_{i \in \mathcal{S}_{\text {num }}} \sum_{m \in \mathcal{M}^{(i)}} \omega^{(i)} \Delta f^{(i)}\left(\alpha_{m}^{(i)} \log \left(\gamma_{m}^{(i)}\left(e^{\tilde{\mathbf{p}}}\right)\right)+\beta_{m}^{(i)}\right) \\
& \text { s.t. } \sum_{i \in \mathcal{S}_{\text {num }}} \sum_{m \in \mathcal{M}^{(i)}} e^{\tilde{p}_{m}^{(i)}} \leq P_{0} .
\end{aligned}
$$

Expanding the term of $\log \left(\gamma_{m}^{(i)}\left(e^{\tilde{\mathbf{p}}}\right)\right)$ yields the following expression

$$
\begin{aligned}
& \log \left(\gamma_{m}^{(i)}\left(e^{\tilde{\mathbf{p}}}\right)\right)=2 \log \left|\Lambda_{m, m}^{(i)}\right|+\tilde{p}_{m}^{(i)} \\
& -\left(\sum_{j \in \mathcal{S}_{\text {num }} /\{i\}} \sum_{n=0}^{\left\lceil\frac{\nu^{(j)}}{\nu(i)}\right\rceil N_{c}^{(j)}-1}\left|\Psi_{k, m, n}^{(i \leftarrow j)}\right|^{2} e^{\left\{\tilde{p}_{n \bmod N_{c}^{(j)}}^{(j)}\right\}}\right. \\
& \left.\quad+\left|\hat{w}_{k, m}^{(i)}\right|^{2}\right)
\end{aligned}
$$

which comprises a sum of a linear term and a convex log-sumexp term. This proves the convexity of the objective function in (59). The constraint function, expressed as a sum of convex terms $\left(e^{\tilde{u}_{m}^{(i)}}\right)$, is also convex. The above analysis concludes that (59) is a convex optimization problem. Therefore, it can be solved by using a standard convex optimization package like CVX [36]. Here, we are maximizing a lower bound on the achievable sum rate. The bound can then be improved iteratively, which yields the following procedure.

\section{NUMERICAL RESULTS}

In this section, the impact of several system parameters on the level of distortion is examined through the analytical 

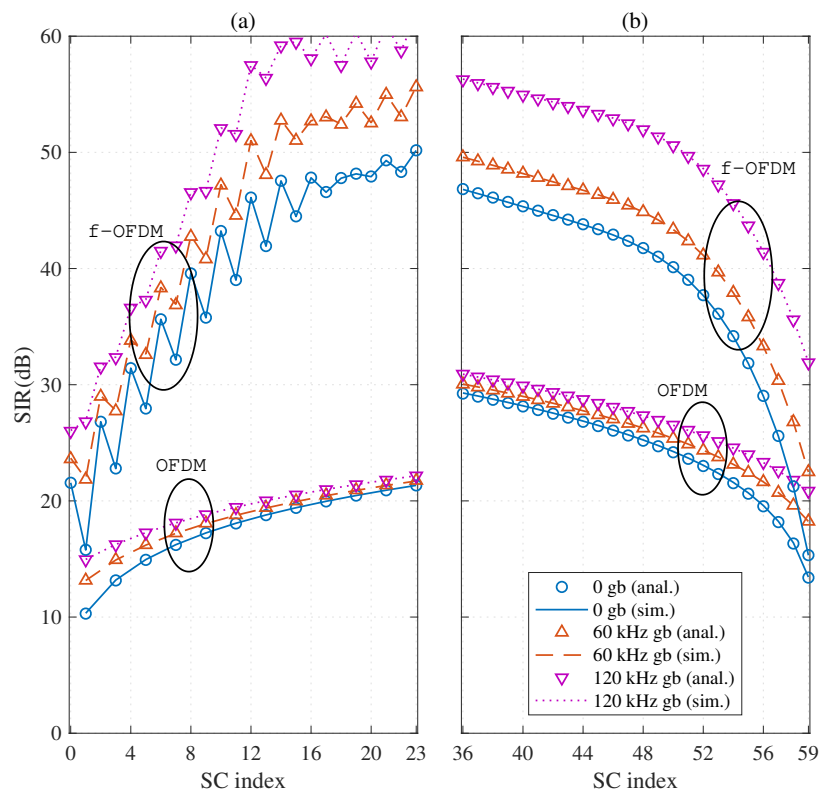

Fig. 4. Inter-NI as a function of the guard band settings on interfering BWPs. TDL-C channel with 300 ns delay spread, the length of all filters: 3 SLs, and no guard band in $\mathrm{BWP}^{\left(a_{0}\right)}$. Axes truncated to show 24 subcarriers to the interference source side, gb refers to guard band. (a) Interference source: signal of $\mathrm{BWP}^{\left(a_{1}\right)}$; (b) Interference source: signal of $\mathrm{BWP}^{\left(a_{2}\right)}$.

studies conducted in Section III and IV. Moreover, the performance of the proposed power allocation scheme in Section $\mathrm{V}$ is also evaluated via Monte Carlo simulations. Table I lists numerology-related parameters. Three BWPs $\left(\mathrm{BWP}^{\left(a_{0}\right)}\right.$, $\left.\mathrm{BWP}^{\left(a_{1}\right)}, \mathrm{BWP}^{\left(a_{2}\right)}\right)$ with different numerologies are considered in a system with total bandwidth of $1024 \times 15 \mathrm{kHz}$. All BWPs are assumed to have 5 physical resource blocks (PRBs) equivalent to 60 subcarriers allocation, and guard bands are allocated within BWPs. The level of distortion and bit error rate (BER) are always evaluated for $\operatorname{BWP}^{\left(a_{0}\right)}$ while signals from $\mathrm{BWP}^{\left(a_{1}\right)}$ and $\mathrm{BWP}^{\left(a_{2}\right)}$ serve as interNI sources. In this setting, signal from $\mathrm{BWP}^{\left(a_{1}\right)}$ with a wider subcarrier spacing interferes with $\mathrm{BWP}^{\left(a_{0}\right)}$ from the left-hand side, while signal from $\mathrm{BWP}^{\left(a_{2}\right)}$ with a narrower subcarrier spacing interferes from the right-hand side. The AWGN noise power density is assumed to be $-174 \mathrm{dBm} / \mathrm{Hz}$. No other interference sources are present and the intraNI is well eliminated by the insertion of the cyclic redundancy. Thus, the term "interference" below refers to "interNI" between different BWPs.

In the case of f-OFDM, soft-truncated sinc filters defined in [37] are implemented at the transmitter and receiver. The roll-off factor of all used time domain window is fixed to 0.6, while different filter lengths are employed to investigate the impact of filters. The length of a filter is measured by the number of sidelobes (SLs) it has on each side. For simplicity, we assume that matched filters are used in all cases except the one which uses unmatched filters to specifically investigate the impact of transparent filtering in Fig. 6.

The SIR expression developed in (48) is evaluated with different settings to study the impact of guard bands and filters
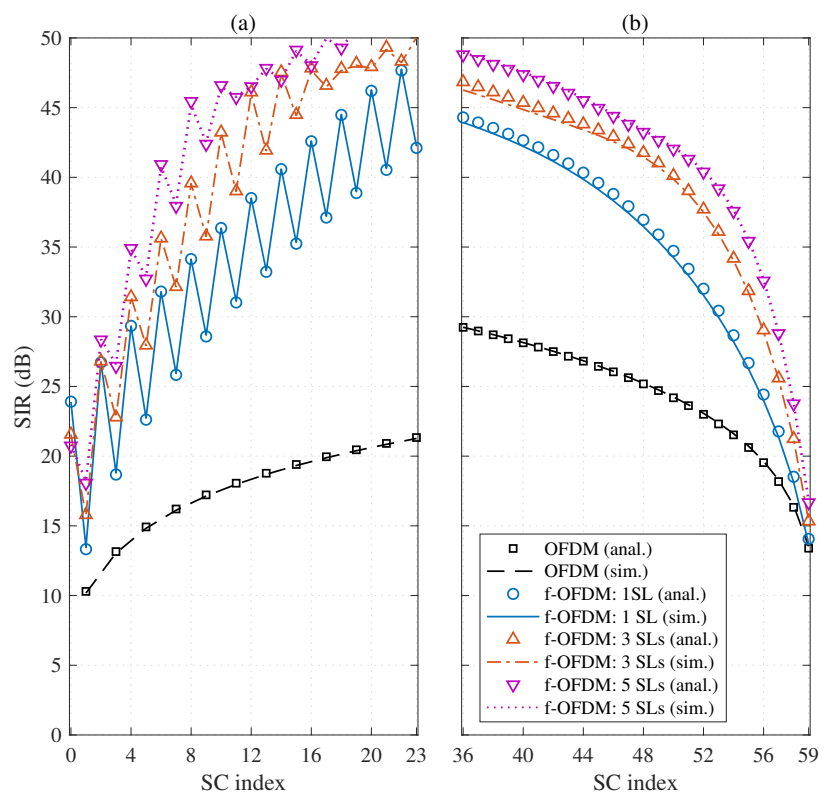

Fig. 5. Inter-NI as a function of the filter length of interfering sources (matched filtering scenario). TDL-C channel with 300 ns delay spread, no guard band. The length of filters in $\operatorname{BWP}^{\left(a_{0}\right)}$ is fixed at $3 \mathrm{SLs}$, while the filters in $\operatorname{BWP}^{\left(a_{1}\right)}$ and $\operatorname{BWP}^{\left(a_{2}\right)}$ take three different lengths: 1 SLs, 3SLs and 5 SLs. Axes truncated to show 24 subcarriers to the interference source side. (a) Interference source: signal of $\mathrm{BWP}^{\left(a_{1}\right)}$; (b) Interference source: signal of $\operatorname{BWP}^{\left(a_{2}\right)}$
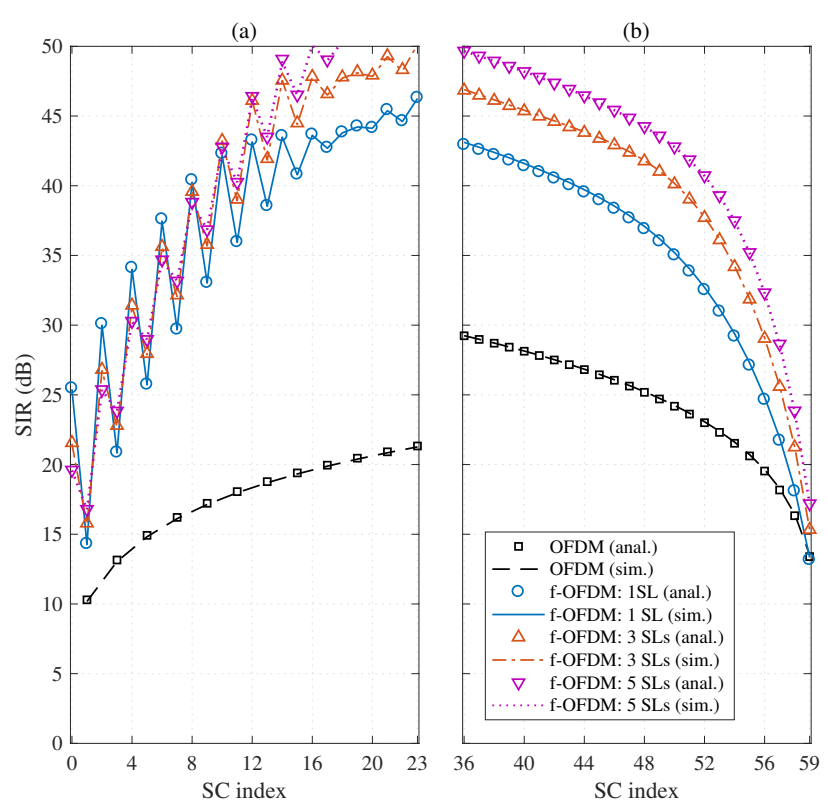

Fig. 6. Inter-NI as a function of the filter length of interfering sources (transparent filtering scenario). TDL-C channel with $300 \mathrm{~ns}$ delay spread, no guard band. The length of filters at the transmitter is fixed at 3 SLs, while the filters at the receiver take three different lengths: 1 SLs, 3SLs and 5 SLs. Axes truncated to show 24 subcarriers to the interference source side. (a) Interference source: signal of $\mathrm{BWP}^{\left(a_{1}\right)}$; (b) Interference source: signal of $\operatorname{BWP}^{\left(a_{2}\right)}$. 
on the interNI under a $300 \mathrm{~ns}$ TDL-C channel defined in [38] in Figs. 4-6. Monte Carlo simulations are conducted to validate our analytical work, and the closely matched results indicate that our derivations are valid. It can be observed from all the three figures that the level of interference decreases (SIR increases) as subcarriers move away from the interference sources. In OFDM, the SIR curves climbs very slowly over the subcarriers, and the SIR finally stays a level at about $20 \mathrm{~dB}$ and $30 \mathrm{~dB}$ with $\mathrm{BWP}^{\left(a_{1}\right)}$, and $\mathrm{BWP}^{\left(a_{2}\right)}$ as the interference source, respectively, which suggests that subcarriers of a numerology suffer more distortion from a source with wider subcarrier spacing. In contrast, the SIR curves of f-OFDM rise much more rapidly over the subcarriers, and the level of interference is more independent of numerology as curves corresponding to different interference source have similar growing rate given the same setting. When comparing the SIR curves between OFDM and f-OFDM, we can easily see that the latter performs much better except the first few subcarriers which only show relatively small improvement. This implies that the level of interference can be controlled below a pre-defined value with the employment of filtering and guard bands.

The impact of guard band on the level of interference in terms of SIR is illustrated in Fig. 4. The two filters at the receiver and transmitter in f-OFDM are assumed to have 3 SLs. No guard band is adopted for $\mathrm{BWP}^{\left(a_{0}\right)}$, while three different guard band sizes $(0,60 \mathrm{kHz}, 120 \mathrm{kHz})$ are considered for interfering BWPs. As the guard band becomes wider, we observe that the level of the interference reduces (SIR increases) for both OFDM and f-OFDM. However, the effect is much more significant in f-OFDM than OFDM for which the SIR improvement becomes marginal as the subcarriers move away from the interference sources. Moreover, when it comes to different numerologies, we found that guard band functions more effectively in terms of reducing interference for the BWP with a smaller subcarrier spacing in OFDM by comparing Fig. 4(a) with Fig. 4(b). However, this trend is not well preserved in $\mathrm{f}$-OFDM due to the additional signal processing.

In Figs. 5 and 6, we study how filters affect the level of interference in the case of matched filtering and transparent filtering, respectively. Fig. 5 shows the SIR variation for $\operatorname{BWP}^{\left(a_{0}\right)}$, when filters of different length in interfering BWPs are considered. Matched filters are assumed for all BWPs, and the length of the filters used in $\operatorname{BWP}^{\left(a_{0}\right)}$ is fixed. It is apparent that the interference decreases as the length of filters in interfering sources increases. This can be well explained by the fact that longer filters enjoy better frequency localization. Fig. 6 depicts the impact on the interference from transparent filtering (unmatched filters between the receiver and the transmitter of all three BWPs). The length of the filters at the transmitters are configured at 3SLs, while the receivers have three different settings on filter length: 1SLs, 3SLs, and 5SLs. When the filter length increases, we see the similar trend as in the matched filtering case, which confirms the feasibility of transparent filtering.

Fig. 7 and Fig. 8 show BER performance of BWP ${ }^{\left(a_{0}\right)}$ under different settings on power offset with 16-QAM modulation under the AWGN and TDL-C channel, respectively. We notice that the dashed curves of the two figures, corresponding to
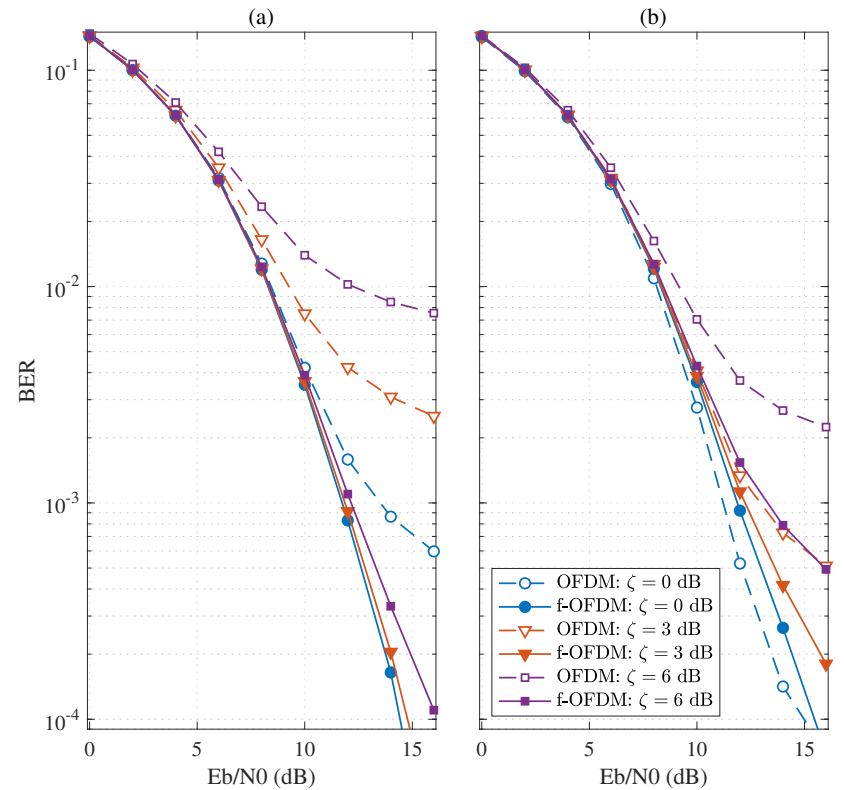

Fig. 7. BER performance with different settings on power offset under AWGN channel and 16-QAM modulation scheme. One guard subcarrier is implemented on each side of all three BWPs. Power offset factor $\zeta$ is defined as $\zeta=\left(\frac{\sigma^{\left(a_{i}\right)}}{\sigma^{\left(a_{0}\right)}}\right)^{2} \mathrm{~dB}, i=1,2$. (a) Interference source: signal of $\operatorname{BWP}^{\left(a_{1}\right)}$; (b) Interference source: signal of $\mathrm{BWP}^{\left(a_{2}\right)}$.

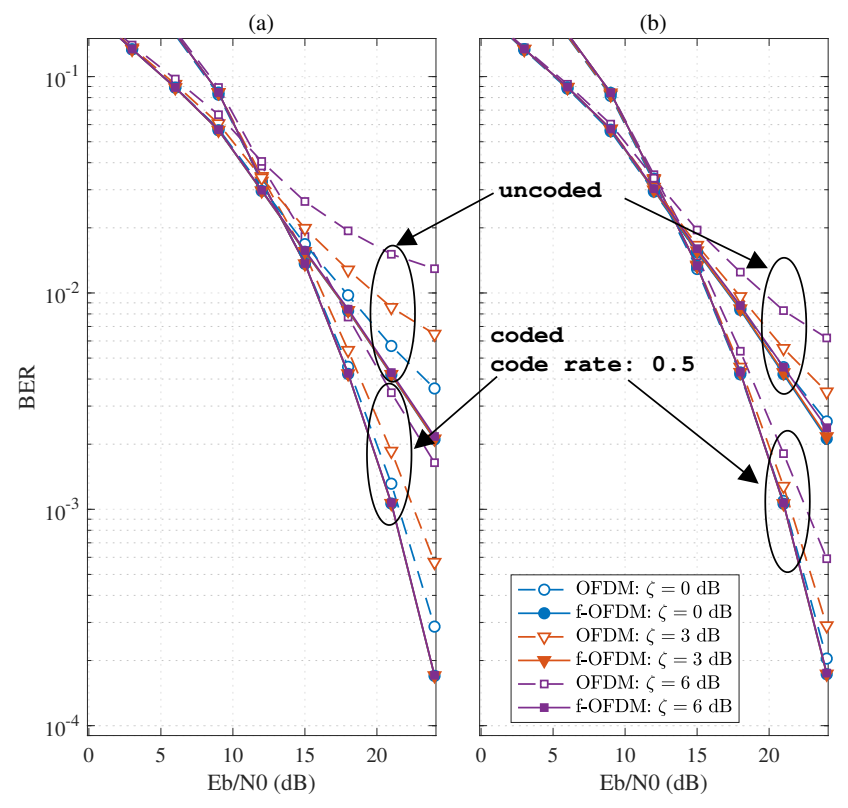

Fig. 8. BER performance with different settings on power offset under 300 ns TDL-C channel and 16-QAM modulation scheme. One guard subcarrier is implemented on each side of all three BWPs. Power offset factor $\zeta$ is defined as $\zeta=\left(\frac{\sigma^{\left(a_{i}\right)}}{\sigma^{\left(a_{0}\right)}}\right)^{2} \mathrm{~dB}, i=1,2$. (a) Interference source: signal of $\mathrm{BWP}^{\left(a_{1}\right)}$; (b) Interference source: signal of $\operatorname{BWP}^{\left(a_{2}\right)}$. 
different power offset in OFDM, are well apart from each other, and error floors quickly develop when power offset is high especially for the uncoded signal. The significant BER degradation caused by each $3 \mathrm{~dB}$ increase in power offset suggests that power offset has a significant impact on the interference between different numerologies in OFDM. In contrast, the BER degradation due to power offset is much lower in f-OFDM for both the models. This implies that $\mathrm{f}$ OFDM systems are more resilient to power offset than OFDM systems, which again conforms the importance of the spectrum confinement techniques to the multi-numerology system. When comparing interference from different numerologies between Fig. 7 (a) and (b), one can observe that $\mathrm{BWP}^{\left(a_{0}\right)}$ suffers more interference from the source with a wider subcarrier spacing in the presence of power offset.

The spectrum efficiency (SE) of the proposed power allocation in Section V is numerically compared with the other two schemes, IWF and equal power allocation, in f-OFDM/OFDM multi-numerology systems in Fig. 9. The TDL-C channel with $300 \mathrm{~ns}$ delay spread is considered. Without loss of generality, we assume all BWPs are equally weighed and no guard band is used. We observe that the SE of all schemes are very close in Fig. 9 (a), while the SE of the proposed power allocation method stands out from the others in Fig. 9 (b). This concludes that power allocation works more effectively in higher SNR region where the transmission tends to be interference-limited. It is worth mentioning that f-OFDM performs generally better than OFDM in all schemes. However, the SE improvement with the proposed scheme is higher in OFDM. This can be explained by the fact that there is more interNI in OFDM; Thus, there is more room for improvement through power allocation.

\section{CONCLUSION}

This paper developed a generic OFDM/f-OFDM system model in the presence of mixed numerologies, which complies with the filter transparency requirement by 3GPP. With the developed model, the interference between different numerologies was analyzed. The analytical metric for quantifying the level of the distortion was then derived as a function of several system parameters. The impact of these parameters on the inter-numerology interference was investigated analytically and numerically. A case study on optimizing power allocation based on the derived analytical model was also presented. The work presented in this paper provided an analytical guidance on the system design in support of $5 \mathrm{G}$ multi-service transmissions over a unified physical infrastructure.

\section{ACKNOWLEDGMENT}

The authors would like to acknowledge the support of the University of Surrey 5GIC (http://www.surrey.ac.uk/5gic) members for this work.

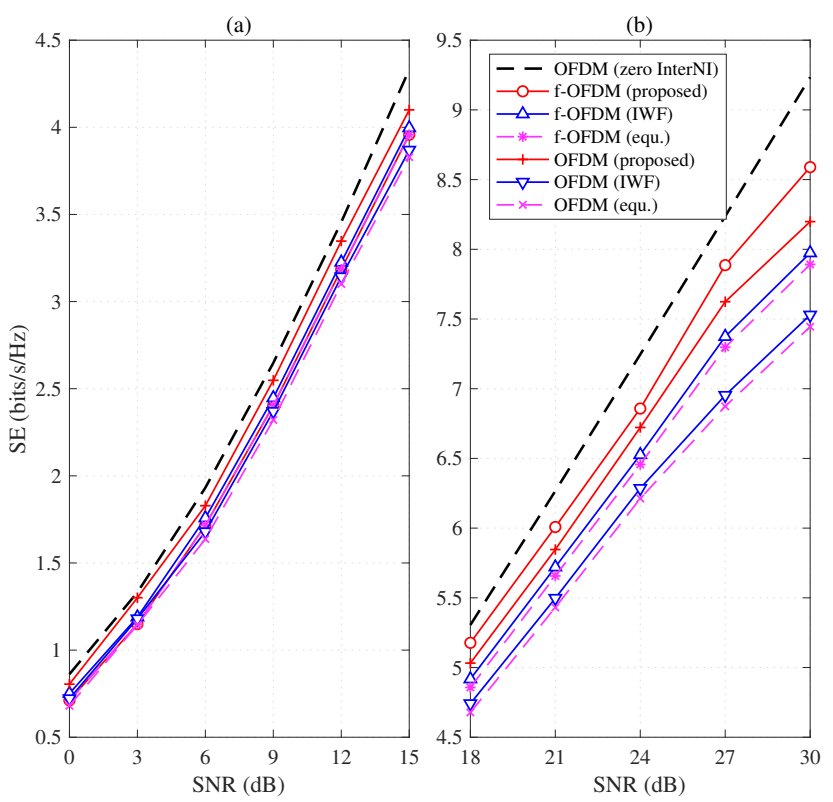

Fig. 9. Spectrum efficiency comparison among different power allocation schemes under $300 \mathrm{~ns}$ TDL-C channel in the presence of mixed numerologies: proposed, IWF, and equal power allocation. (a) Low SNR region; (b) High SNR region.

\section{APPENDIX A}

\section{PROOF OF PROPOSITION 1 AND PROPOSITION 2}

As shown in Fig. 3, the length of symbols is different across numerologies. The symbol length (including CR) of the $i$-th numerology can be calculated as

$$
L^{(i)}=\frac{L_{\text {ref }}}{\nu^{(i)}},
$$

where $L_{\text {ref }}$ is the symbol length corresponding to $15 \mathrm{kHz}$ subcarrier spacing. The $k$-th symbol of the $i$-th numerology spans in the time interval $\left(k \frac{L_{\text {ref }}}{\nu^{(i)}},(k+1) \frac{L_{\text {ref }}}{\nu^{(i)}}\right)$.

When the $i$-th numerology is multiplexed with the $j$-th numerology, the later has longer or shorter symbol length depending on its subcarrier spacing.

If $j \in \mathcal{S}_{\text {num }}^{(i-)}$, i.e., the $j$-th numerology has a narrower subcarrier spacing and a longer symbol length. Therefore, the signal in the time interval $\left(k \frac{L_{\text {ref }}}{\nu^{(i)}},(k+1) \frac{L_{\text {ref }}}{\nu^{(i)}}\right)$ occupies only a portion of a OFDM window of the $j$-th numerology, and the index of the window can be calculated as

$$
\left\lfloor\frac{k L_{\mathrm{ref}} / \nu^{(i)}}{L_{\mathrm{ref}} / \nu^{(j)}}\right\rfloor=\left\lfloor k \frac{\nu^{(j)}}{\nu^{(i)}}\right\rfloor .
$$

Moreover, the symbol duration of the $j$-th numerology is $\frac{\nu^{(i)}}{\nu^{(j)}}$ times longer than that of the $i$-th numerology. If we equally divide each OFDM symbol of the $j$-th numerology into $\frac{\nu^{(i)}}{\nu^{(j)}}$ symbol parts (SPs), then exactly one of them fits in the time interval $\left(k \frac{L_{\text {ref }}}{\nu^{(i)}},(k+1) \frac{L_{\text {ref }}}{\nu^{(i)}}\right)$, and it can be found as

$$
\begin{aligned}
\left(k \frac{\nu^{(j)}}{\nu^{(i)}}-\left\lfloor k \frac{\nu^{(j)}}{\nu^{(i)}}\right\rfloor\right) \frac{\nu^{(i)}}{\nu^{(j)}} & =k-\left\lfloor\frac{k}{\nu^{(i)} / \nu^{(j)}}\right\rfloor\left(\nu^{(i)} / \nu^{(j)}\right) \\
& =k \bmod \left(\nu^{(i)} / \nu^{(j)}\right) .
\end{aligned}
$$


To sum up, the $\left(k \bmod \frac{\nu^{(i)}}{\nu^{(j)}}\right)$-th SP of the $\left(\left\lfloor k \frac{\nu^{(j)}}{\nu^{(i)}}\right\rfloor\right)$-th OFDM symbol of the $j$-th numerology overlaps with $k$-th OFDM symbol of the $i$-th numerology if $j \in \mathcal{S}_{\text {num }}^{(i-)}$.

In contrast, if $j \in \mathcal{S}_{\text {num }}^{(i+)}$, each symbol length of the $i$-th numerology equals the sum of corresponding $\frac{\nu^{(j)}}{\nu^{(i)}}$ symbols of the $j$-th numerology. The index of the first symbol in the time interval $\left(k \frac{L_{\text {ref }}}{\nu^{(i)}},(k+1) \frac{L_{\text {ref }}}{\nu^{(i)}}\right)$ can be calculated as

$$
\frac{k L_{\mathrm{ref}} / \nu^{(i)}}{L_{\mathrm{ref}} / \nu^{(j)}}=k \frac{\nu^{(j)}}{\nu^{(i)}}
$$

and the index of the last one can be obtained as

$$
\frac{(k+1) L_{\mathrm{ref}} / \nu^{(i)}}{L_{\mathrm{ref}} / \nu^{(j)}}-1=(k+1) \frac{\nu^{(j)}}{\nu^{(i)}}-1 .
$$

Therefore, the $k$-th symbol of the $i$-th numerology overlaps with $\frac{\nu^{(j)}}{\nu^{(i)}}$ symbols of the $j$-th numerology in the range of $\frac{k \nu^{(j)}}{\nu^{(i)}}, \frac{k \nu^{(j)}}{\nu^{(i)}}+1, \cdots, \frac{(k+1) \nu^{(j)}}{\nu^{(i)}}-1$ if $j \in \mathcal{S}_{\text {num }}^{(i+)}$.

\section{APPENDiX B}

\section{DERIVATION OF $\mathbf{z}_{k}^{(i)}$}

Substituting the expression of $\mathbf{r}_{k}^{(i)}$ in (21) into (25), we obtain

$$
\begin{aligned}
\mathbf{z}_{k}^{(i)} & =\mathbf{V}^{(i: u)} \mathbf{H}^{(i: u)} \mathbf{s}_{k-2}^{(i)}+\left(\mathbf{V}^{(i: u)} \mathbf{H}^{(i: o)}+\mathbf{V}^{(i: o)} \mathbf{H}^{(i: u)}\right) \mathbf{s}_{k-1}^{(i)} \\
& +\left(\mathbf{V}^{(i: o)} \mathbf{H}^{(i: o)}+\mathbf{V}^{(i: l)} \mathbf{H}^{(i: u)}\right) \mathbf{s}_{k}^{(i)}+\mathbf{V}^{(i: l)} \mathbf{H}^{(i: o)} \mathbf{s}_{k+1}^{(i)}
\end{aligned}
$$

Since the multiplication of two strict upper triangular matrices results in a zero matrix, the term $\mathbf{V}^{(i: u)} \mathbf{H}^{(i: u)} \mathbf{s}_{k-2}^{<i>}$ can be canceled. Replacing $\mathbf{s}_{k}^{(i)}$ of the above equation with its expression in (7), followed by merging of similar items with respect to $\mathbf{x}_{k}^{(i)}$, yields

$$
\begin{aligned}
\mathbf{z}_{k}^{(i)} & =\mathbf{V}^{(i: u)} \mathbf{H}^{(i: o)} \mathbf{U}^{(i: u)} \mathbf{x}_{k-2}^{(i)}+\left(\mathbf{V}^{(i: u)} \mathbf{H}^{(i: o)} \mathbf{U}^{(i: o)}\right. \\
& +\mathbf{V}^{(i: o)} \mathbf{H}^{(i: u)} \mathbf{U}^{(i: o)}+\mathbf{V}^{(i: o)} \mathbf{H}^{(i: o)} \mathbf{U}^{(i: u)} \\
& \left.+\mathbf{V}^{(i: l)} \mathbf{H}^{(i: u)} \mathbf{U}^{(i: u)}\right) \mathbf{x}_{k-1}^{(i)}+\left(\mathbf{V}^{(i: u)} \mathbf{H}^{(i: o)} \mathbf{U}^{(i: l)}\right. \\
& +\mathbf{V}^{(i: o)} \mathbf{H}^{(i: u)} \mathbf{U}^{(i: l)}+\mathbf{V}^{(i: o)} \mathbf{H}^{(i: o)} \mathbf{U}^{(i: o)} \\
& \left.+\mathbf{V}^{(i: l)} \mathbf{H}^{(i: o)} \mathbf{U}^{(i: u)}\right) \mathbf{x}_{k}^{(i)}+\left(\mathbf{V}^{(i: o)} \mathbf{H}^{(i: o)} \mathbf{U}^{(i: l)}\right. \\
& \left.+\mathbf{V}^{(i: l)} \mathbf{H}^{(i: u)} \mathbf{U}^{(i: l)}+\mathbf{V}^{(i: l)} \mathbf{H}^{(i: o)} \mathbf{U}^{(i: o)}\right) \mathbf{x}_{k+1}^{(i)} \\
& +\mathbf{V}^{(i: l)} \mathbf{H}^{(i: o)} \mathbf{U}^{(i: l)} \mathbf{x}_{k+2}^{(i)} .
\end{aligned}
$$

It is proved in Appendix D that $\mathbf{U}^{(i: u)} \mathbf{H}^{(i: o)}$ is a strictly upper triangular matrix, and $\mathbf{V}^{(i: l)} \mathbf{H}^{(i: o)}$ is a strictly lower triangular matrix. As the multiplication of two strictly upper/lower triangular matrices equals a zero matrix, we have $\mathbf{V}^{(i: u)} \mathbf{H}^{(i: o)} \mathbf{U}^{(i: u)}=0, \quad \mathbf{V}^{(i: l)} \mathbf{H}^{(i: o)} \mathbf{U}^{(i: l)}=0$, and $\mathbf{V}^{(i: l)} \mathbf{H}^{(i: u)} \mathbf{U}^{(i: u)}=0 . \mathbf{z}_{k}^{(i)}$ in (67) can then be simplified as

$$
\mathbf{z}_{k}^{(i)}=\boldsymbol{\Theta}_{\mathrm{pre}}^{(i)} \mathbf{x}_{k-1}^{(i)}+\boldsymbol{\Theta}^{(i)} \mathbf{x}_{k}^{(i)}+\boldsymbol{\Theta}_{\text {next }}^{(i)} \mathbf{x}_{k+1}^{(i)},
$$

where

$$
\begin{aligned}
\Theta_{\mathrm{pre}}^{(i)}= & \mathbf{V}^{(i: u)} \mathbf{H}^{(i: o)} \mathbf{U}^{(i: o)} \\
& +\mathbf{V}^{(i: o)} \mathbf{H}^{(i: u)} \mathbf{U}^{(i: o)}+\mathbf{V}^{(i: o)} \mathbf{H}^{(i: o)} \mathbf{U}^{(i: u)} \\
\boldsymbol{\Theta}^{(i)}= & \mathbf{V}^{(i: u)} \mathbf{H}^{(i: o)} \mathbf{U}^{(i: l)}+\mathbf{V}^{(i: o)} \mathbf{H}^{(i: u)} \mathbf{U}^{(i: l)} \\
& +\mathbf{V}^{(i: o)} \mathbf{H}^{(i: o)} \mathbf{U}^{(i: o)}+\mathbf{V}^{(i: l)} \mathbf{H}^{(i: o)} \mathbf{U}^{(i: u)} \\
\Theta_{\mathrm{next}}^{(i)}= & \mathbf{V}^{(i: o)} \mathbf{H}^{(i: o)} \mathbf{U}^{(i: l)} \\
& +\mathbf{V}^{(i: l)} \mathbf{H}^{(i: u)} \mathbf{U}^{(i: l)}+\mathbf{V}^{(i: l)} \mathbf{H}^{(i: o)} \mathbf{U}^{(i: o)}
\end{aligned}
$$

\section{APPENDIX C DERIVATION OF $\tilde{\mathbf{z}}_{k}^{(i)}$}

Substituting the expression of $\mathbf{r}_{k}^{(i)}$ in (21) into (26) yields

$$
\begin{aligned}
& \tilde{\mathbf{z}}_{k}^{(i)}=\left(\mathbf{V}^{(i: u)} \mathbf{H}^{(i: o)}+\mathbf{V}^{(i: o)} \mathbf{H}^{(i: u)}\right) \tilde{\mathbf{s}}_{k-1}^{(i)} \\
& \quad+\left(\mathbf{V}^{(i: o)} \mathbf{H}^{(i: o)}+\mathbf{V}^{(i: l)} \mathbf{H}^{(i: u)}\right) \tilde{\mathbf{s}}_{k}^{(i)}+\mathbf{V}^{(i: l)} \mathbf{H}^{(i: o)} \tilde{\mathbf{s}}_{k+1}^{(i)}
\end{aligned}
$$

According to Eq. (15), the vector $\tilde{\mathbf{s}}_{k}^{(i)}$ is the mixed signal from all numerologies except the $i$-th one. Thus, we can express $\tilde{\mathbf{z}}_{k}^{(i)}$ as a sum of the signal from those numerologies as

$$
\tilde{\mathbf{z}}_{k}^{(i)}=\sum_{j \in \mathcal{S}_{\text {num } \backslash\{i\}}} \mathbf{z}_{k}^{(i \leftarrow j)}
$$

where $\mathbf{z}_{k}^{(i \leftarrow j)}$ can be interpreted as the signal from the $j$-th numerology which lies in the $k$-th OFDM window of the $i$-th numerology. Based on (69), it can be expressed as

$$
\begin{aligned}
\mathbf{z}_{k}^{(i \leftarrow j)} & =\left(\mathbf{V}^{(i: u)} \mathbf{H}^{(i: o)}+\mathbf{V}^{(i: o)} \mathbf{H}^{(i: u)}\right) \mathbf{s}_{k-1}^{(i \leftarrow j)} \\
& +\left(\mathbf{V}^{(i: o)} \mathbf{H}^{(i: o)}+\mathbf{V}^{(i: l)} \mathbf{H}^{(i: u)}\right) \mathbf{s}_{k}^{(i \leftarrow j)} \\
& +\mathbf{V}^{(i: l)} \mathbf{H}^{(i: o)} \mathbf{s}_{k+1}^{(i \leftarrow j)}
\end{aligned}
$$

where the definition of $\mathbf{s}_{k}^{(i \leftarrow j)}$ is given in Proposition 1 and Proposition 2 for $j \in \mathcal{S}_{\text {num }}^{(i-)}$ and $j \in \mathcal{S}_{\text {num }}^{(i+)}$, respectively. In the following, we will further expand $\mathbf{z}_{k}^{(i \leftarrow j)}$ for $j \in \mathcal{S}_{\text {num }}^{(i-)}$ and $j \in \mathcal{S}_{\text {num }}^{(i+)}$, respectively.

\section{A. In the case of $j \in \mathcal{S}_{\text {num }}^{i-}$}

After some basic algebraic manipulations based on (7) and the expression of $\mathbf{s}_{k}^{(i \leftarrow j)}$ given in Proposition 1, we can express $\mathbf{z}_{k}^{(i \leftarrow j)}$ in (71) as a sum of a dominant term and many trivial terms as

$$
\mathbf{z}_{k}^{(i \leftarrow j)}=\underbrace{\mathbf{V}^{(i: o)} \mathbf{H}^{(i: o)} \mathbf{C}_{k}^{(i \leftarrow j)} \mathbf{U}^{(j: o)} \mathbf{x}_{k}^{(i \leftarrow j)}}_{\text {dominant term }}+\underbrace{\boldsymbol{\epsilon}_{k}^{(i \leftarrow j)}}_{\text {trivial terms }},
$$


with

$$
\begin{aligned}
\boldsymbol{\epsilon}_{k}^{(i \leftarrow j)}=\left(\mathbf{V}^{(i: u)} \mathbf{H}^{(i: o)} \mathbf{C}_{k-1}^{(i \leftarrow j)} \mathbf{U}^{(j: u)}\right. & \left.+\mathbf{V}^{(i: o)} \mathbf{H}^{(i: u)} \mathbf{C}_{k-1}^{(i \leftarrow j)} \mathbf{U}^{(j: u)}\right) \underline{\mathbf{x}}_{k-1}^{(i \leftarrow j)} \\
+ & \left(\mathbf{V}^{(i: u)} \mathbf{H}^{(i: o)} \mathbf{C}_{k-1}^{(i \leftarrow j)} \mathbf{U}^{(j: o)}\right. \\
& \left.+\mathbf{V}^{(i: o)} \mathbf{H}^{(i: u)} \mathbf{C}_{k-1}^{(i \leftarrow j)} \mathbf{U}^{(j: o)}\right) \underline{\mathbf{x}}_{k}^{(i \leftarrow j)} \\
+ & \left(\mathbf{V}^{(i: u)} \mathbf{H}^{(i: o)} \mathbf{C}_{k-1}^{(i \leftarrow j)} \mathbf{U}^{(j: l)}\right. \\
& \left.+\mathbf{V}^{(i: o)} \mathbf{H}^{(i: u)} \mathbf{C}_{k-1}^{(i \leftarrow j)} \mathbf{U}^{(j: l)}\right) \underline{\mathbf{x}}_{k+1}^{(i \leftarrow j)} \\
+ & \left(\mathbf{V}^{(i: o)} \mathbf{H}^{(i: o)} \mathbf{C}_{k}^{(i \leftarrow j)} \mathbf{U}^{(j: u)}\right. \\
& \left.+\mathbf{V}^{(i: l)} \mathbf{H}^{(i: u)} \mathbf{C}_{k}^{(i \leftarrow j)} \mathbf{U}^{(j: u)}\right) \mathbf{x}_{k-1}^{(i \leftarrow j)} \\
+ & \left(\mathbf{V}^{(i: o)} \mathbf{H}^{(i: o)} \mathbf{C}_{k}^{(i \leftarrow j)} \mathbf{U}^{(j: l)}\right. \\
& \left.+\mathbf{V}^{(i: l)} \mathbf{H}^{(i: u)} \mathbf{C}_{k}^{(i \leftarrow j)} \mathbf{U}^{(j: l)}\right) \mathbf{x}_{k+1}^{(i \leftarrow j)} \\
+ & \mathbf{V}^{(i: l)} \mathbf{H}^{(i: u)} \mathbf{C}_{k}^{(i \leftarrow j)} \mathbf{U}^{(j: o)} \mathbf{x}_{k}^{(i \leftarrow j)} \\
& +\mathbf{V}^{(i: l)} \mathbf{H}^{(i: o)} \mathbf{C}_{k+1}^{(i \leftarrow j)} \mathbf{U}^{(j: u)} \overline{\mathbf{x}}_{k-1}^{(i \leftarrow j)} \\
+ & \mathbf{V}^{(i: l)} \mathbf{H}^{(i: o)} \mathbf{C}_{k+1}^{(i \leftarrow j)} \mathbf{U}^{(j: o)} \overline{\mathbf{x}}_{k}^{(i \leftarrow j)} \\
& +\mathbf{V}^{(i: l)} \mathbf{H}^{(i: o)} \mathbf{C}_{k+1}^{(i \leftarrow j)} \mathbf{U}^{(j: l)} \overline{\mathbf{x}}_{k+1}^{(i \leftarrow j)}
\end{aligned}
$$

where $\mathbf{x}_{k}^{(i \leftarrow j)}=\mathbf{x}_{\left\lceil k \frac{\nu(j)}{\nu^{(i)}}\right\rceil}^{(j)}, \underline{\mathbf{x}}_{k}^{(i \leftarrow j)}=\mathbf{x}_{\left\lceil(k-1) \frac{\nu^{(j)}}{\nu(i)}\right\rceil}^{(j)}$, and $\overline{\mathbf{x}}_{k}^{(i \leftarrow j)}=\mathbf{x}_{\left\lceil(k+1) \frac{\nu^{(j)}}{\nu^{(i)}}\right\rceil}^{(j)}$.

As the trivial terms correspond to filter and channel spreadings, and their energy is significantly less than that of the dominant part of the signal. Moreover, CR is assumed to be sufficiently long to capture the main lobe of the filters and the channel spreading. Thus, the residual spreading can be ignored, and we can approximate $\mathbf{z}_{k}^{(i \leftarrow j)}$ as

$$
\mathbf{z}_{k}^{(i \leftarrow j)} \approx \mathbf{V}^{(i: o)} \mathbf{H}^{(i: o)} \mathbf{C}_{k}^{(i \leftarrow j)} \mathbf{U}^{(j: o)} \mathbf{x}_{k}^{(i \leftarrow j)}, j \in \mathcal{S}_{\text {num }}^{(i-)} .
$$

\section{B. In the case of $j \in \mathcal{S}_{\text {num }}^{(i+)}$}

Based on (7) and the expression of $\mathbf{s}_{k}^{(i \leftarrow j)}$ given in Proposition 2, following the similar procedure in $\mathrm{A}\left(j \in \mathcal{S}_{\text {num }}^{(i-)}\right)$, we can approximate $\mathbf{z}_{k}^{(i \leftarrow j)}\left(j \in \mathcal{S}_{\text {num }}^{(i+)}\right)$ as

$$
\begin{array}{r}
\mathbf{z}_{k}^{(i \leftarrow j)} \approx \mathbf{V}^{(i: o)} \mathbf{H}^{(i: o)} \operatorname{blkdiag}\left(\mathbf{U}^{(j: o)}, \frac{\nu^{(j)}}{\nu^{(i)}}\right) \mathbf{x}_{k}^{(i \leftarrow j)}, \\
j \in \mathcal{S}_{\text {num }}^{(i+)} .
\end{array}
$$

where

$$
\mathbf{x}_{k}^{(i \leftarrow j)}=\left[\left(\mathbf{x}_{\frac{k \nu(j)}{\nu(i)}}^{(j)}\right)^{T},\left(\mathbf{x}_{\frac{k \nu(j)}{\nu(i)}+1}^{(j)}\right)^{T}, \cdots,\left(\mathbf{x}_{\frac{(k+1) \nu(j)}{\nu(i)}-1}^{(j)}\right)^{T}\right]^{T} .
$$

Combining $\mathbf{z}_{k}^{(i \leftarrow j)}$ for $j \in \mathcal{S}_{\text {num }}^{(i-)}$ and $j \in \mathcal{S}_{\text {num }}^{(i+)}$, we finally obtain

$$
\begin{aligned}
\tilde{\mathbf{z}}_{k}^{(i)} & \approx \sum_{j \in \mathcal{S}_{\text {num }} \backslash\{i\}} \mathbf{z}_{k}^{(i \leftarrow j)} \\
& =\sum_{j \in \mathcal{S}_{\text {num }}^{(i-)}} \mathbf{V}^{(i: o)} \mathbf{H}^{(i: o)} \mathbf{C}_{k}^{(i \leftarrow j)} \mathbf{U}^{(j: o)} \mathbf{x}_{k}^{(i \leftarrow j)} \\
& +\sum_{j \in \mathcal{S}_{\text {num }}^{(i+)}} \mathbf{V}^{(i: o)} \mathbf{H}^{(i: o)} \operatorname{blkdiag}\left(\mathbf{U}^{(i: o)}, \frac{\nu^{(j)}}{\nu^{(i)}}\right) \mathbf{x}_{k}^{(i \leftarrow j)}
\end{aligned}
$$

\section{APPENDIX D}

\section{THE PROOF OF STRICTLY TRIANGULAR MATRICES}

The product of $\mathbf{U}^{(i: u)} / \mathbf{V}^{(i: u)}$ and $\mathbf{H}^{(i: o)}$ is a strictly upper triangular matrix, such as $D_{r, c}^{(i)}=0,0 \leq \forall r, \forall c \leq L^{(i)}$, when $r>c-\left[L^{(i)}-\frac{N_{u}^{(i)}}{2}-\left(N_{\mathrm{ch}}^{(i)}-1\right)\right]$. As $\mathbf{U}^{(i: u)}$ and $\mathbf{V}^{(i: u)}$ are matrices with the same structure, we only provide detailed steps to prove one of them, the other one can be conducted in the similar fashion.

$$
\begin{aligned}
D_{r, c}^{(i)} & =\sum_{k=1}^{L^{(i)}} U_{r, k}^{(i: u)} H_{k, c}^{(i: o)} \\
& =\sum_{k=1}^{c+N_{\mathrm{ch}}^{(i)}-1} U_{r, k}^{(i: u)} H_{k, c}^{(i: o)}+\sum_{k=c+N_{\mathrm{ch}}^{(i)}}^{L^{(i)}} U_{r, k}^{(i: u)} H_{k, c}^{(i: o)} .
\end{aligned}
$$

Based on the condition $r>c-\left[L^{(i)}-\frac{N_{u}^{(i)}}{2}-\left(N_{\mathrm{ch}}^{(i)}-1\right)\right]$, when $1 \leq k \leq c+N_{c h}^{(i)}-1$, we have

$$
\begin{aligned}
k-\left(L^{(i)}-\frac{N_{u}^{(i)}}{2}\right) & \leq k \\
& \leq c+N_{c h}^{(i)}-1-\left(L^{(i)}-\frac{N_{u}^{(i)}}{2}\right)<r \\
& \Rightarrow k<r+L^{(i)}-\frac{N_{u}^{(i)}}{2},
\end{aligned}
$$

and this gives $U_{r, k}^{(i: u)}=0$ according to (8). Therefore, the first term of (77), $\sum_{k=1}^{c+N_{\mathrm{ch}}^{(i)}-1} U_{r, k}^{(i: u)} H_{k, c}^{(i: o)}=0$.

When $c+N_{\mathrm{ch}}^{(i)} \leq k \leq L^{(i)}$, we have $H_{k, c}^{(i: o)}=0$ based on (18), then the second term of (77), $\sum_{k=j+N_{\mathrm{ch}}^{(i)}}^{L^{(i)}} U_{r, k}^{(i: u)} H_{k, c}^{(i: o)}=0$, is also proved. Therefore, $D_{r, c}^{(i)}=0,0 \leq \forall r, \forall c \leq L^{(i)}$ is proved, because both its sum terms in (77) are zero, when $r>c-\left[L^{(i)}-\frac{N_{u}^{(i)}}{2}-\left(N_{\mathrm{ch}}^{(i)}-1\right)\right]$.

Following a similar procedure, we can prove that the product of $\mathbf{U}^{(i: l)} / \mathbf{V}^{(i: l)}$ and $\mathbf{H}^{(i: o)}$ is a strictly lower triangular matrix with $D_{r, c}^{(i)}=0,0 \leq \forall r, \forall c \leq$ $L^{(i)}$, when $r>c-\left[L^{(i)}-\frac{N_{u}^{(i)}}{2}-\left(N_{\mathrm{ch}}^{(i)}-1\right)\right]$.

\section{REFERENCES}

[1] 3GPP, "Study on Scenarios and Requirements for Next Generation Access Technologies," 3rd Generation Partnership Project, TS 38.9131, 082017.

[2] A. A. Zaidi, R. Baldemair, H. Tullberg et al., "Waveform and Numerology to Support 5G Services and Requirements," IEEE Commun. Mag., vol. 54, no. 11, pp. 90-98, 2016.

[3] P. Banelli, S. Buzzi, G. Colavolpe et al., "Modulation Formats and Waveforms for 5G Networks: Who Will be the Heir of OFDM?: An Overview of Alternative Modulation Schemes for Improved Spectral Efficiency," IEEE Signal Process. Mag., vol. 31, no. 6, pp. 80-93, 2014.

[4] G. Wunder, P. Jung, M. Kasparick et al., "5GNOW: Non-Orthogonal, Asynchronous Waveforms for Future Mobile Applications," IEEE Commun. Mag., vol. 52, no. 2, pp. 97-105, Feb. 2014.

[5] 3GPP, "NR; Physical Channels and Modulation," 3rd Generation Partnership Project, TS 38.211, 092018. 
[6] L. Zhang, A. Ijaz, P. Xiao, and R. Tafazolli, "Multi-Service System: An Enabler of Flexible 5G Air Interface," IEEE Commun. Mag, vol. 55, no. 10, pp. 152-159, 2017.

[7] A. Yazar and H. Arslan, "A Flexibility Metric and Optimization Methods for Mixed Numerologies in 5G and Beyond," IEEE Access, vol. 6, pp. 3755-3764, 2018

[8] L. Zhang, A. Ijaz, P. Xiao et al., "Subband Filtered Multi-Carrier Systems for Multi-Service Wireless Communications," IEEE Trans. Wireless Commun., vol. 16, no. 3, pp. 1893-1907, 2017.

[9] M. Bellanger, D. Le Ruyet, D. Roviras et al., "FBMC Physical Layer: A Primer," PHYDYAS, January, vol. 25, no. 4, pp. 7-10, 2010.

[10] L. Zhang, P. Xiao, A. Zafar, A. ul Quddus, and R. Tafazolli, "FBMC System: An Insight Into Doubly Dispersive Channel Impact," IEEE Trans. Veh. Technol., vol. 66, no. 5, pp. 3942-3956, 2017.

[11] N. Michailow, M. Matthé, I. S. Gaspar et al., "Generalized Frequency Division Multiplexing for 5th Generation Cellular Networks," IEEE Trans. Veh. Commun., vol. 62, no. 9, pp. 3045-3061, 2014.

[12] P. Achaichia, M. Le Bot, and P. Siohan, "Windowed OFDM Versus OFDM/OQAM: A Transmission Capacity Comparison in the HomePlug AV Context," in Proc. IEEE International Symposium on Power Line Communications and Its Applications (ISPLC), 2011, pp. 405-410.

[13] R. Zayani, Y. Medjahdi, H. Shaiek, and D. Roviras, "WOLA-OFDM: A Potential Candidate for Asynchronous 5G," in Proc. IEEE Globecom Workshops (GC Wkshps), 2016, pp. 1-5.

[14] V. Vakilian, T. Wild, F. Schaich et al., "Universal-Filtered Multi-carrier Technique for Wireless Systems Beyond LTE," in Proc. IEEE Globecom Workshops (GC Wkshps), 2013, pp. 223-228.

[15] J. Abdoli, M. Jia, and J. Ma, "Filtered OFDM: A New Waveform for Future Wireless Systems," in Proc. IEEE 16th International Workshop onSignal Processing Advances in Wireless Communications (SPAWC), 2015, pp. 66-70.

[16] L. Zhang, A. Ijaz, P. Xiao et al., "Filtered OFDM Systems, Algorithms, and Performance Analysis for 5G and Beyond," IEEE Trans. Veh. Commun., vol. 66, no. 3, pp. 1205-1218, 2018.

[17] X. Zhang, L. Chen, J. Qiu, and J. Abdoli, "On the Waveform for 5G," IEEE Commun. Mag., vol. 54, no. 11, pp. 74-80, 2016.

[18] S. Wang, J. Armstrong, and J. S. Thompson, "Waveform Performance for Asynchronous Wireless 5G Uplink Communications," in Proc. IEEE 27th Annual International Symposium on Personal, Indoor, and Mobile Radio Communications (PIMRC), 2016, pp. 1-6.

[19] 3GPP, "NR; Base Station (BS) Radio Transmission and Reception," 3rd Generation Partnership Project, TS 38.104, 092019.

[20] T. Levanen, J. Pirskanen, K. Pajukoski, M. Renfors, and M. Valkama, "Transparent Tx and Rx Waveform Processing for 5G New Radio Mobile Communications," IEEE Wirel. Commun., vol. 26, no. 1, pp. 128-136, 2018

[21] M. Benedicks, "On Fourier Transforms of Functions Supported on Sets of Finite Lebesgue Measure," J Math. Anal. Appl., vol. 106, no. 1, pp. 180-183, 1985.

[29] M. Renfors, J. Yli-Kaakinen, T. Levanen, M. Valkama, T. Ihalainen, and J. Vihriala, "Efficient Fast-Convolution Implementation of Filtered
[22] X. Zhang, L. Zhang, P. Xiao et al., "Mixed Numerologies Interference Analysis and Inter-Numerology Interference Cancellation for Windowed OFDM Systems," IEEE Trans. Veh. Technol., 2018.

[23] B. Peköz, S. Köse, and H. Arslan, "Adaptive Windowing of Insufficient CP for Joint Minimization of ISI and ACI Beyond 5G," in Proc. IEEE 28th Annual International Symposium on Personal, Indoor, and Mobile Radio Communications (PIMRC), 2017, pp. 1-5.

[24] A. F. Demir and H. Arslan, "The Impact of Adaptive Guards for 5G and Beyond," in Proc. IEEE 28th Annual International Symposium on Personal, Indoor, and Mobile Radio Communications (PIMRC), 2017, pp. $1-5$.

[25] J. Yli-Kaakinen, T. Levanen, S. Valkonen, K. Pajukoski, J. Pirskanen, M. Renfors, and M. Valkama, "Efficient Fast-Convolution-Based Waveform Processing for 5G Physical Layer," IEEE J. Select. Areas Commun., vol. 35, no. 6, pp. 1309-1326, 2017.

[26] J. Bazzi, K. Kusume, P. Weitkemper, K. Takeda, and A. Benjebbour, "Transparent Spectral Confinement Approach for 5G," in Proc. IEEE 2017 European Conference on Networks and Communications (EuCNC), 2017, pp. 1-5.

[27] P. Guan, D. Wu, T. Tian et al., "5G Field Trials: OFDM-Based Waveforms and Mixed Numerologies," IEEE J. Select. Areas Commun., vol. 35, no. 6, pp. 1234-1243, 2017.

[28] P. Weitkemper, J. Bazzi, K. Kusume et al., "On Regular Resource Grid for Filtered OFDM," IEEE Commun. Lett., vol. 20, no. 12, pp. 24862489, 2016.

CP-OFDM Waveform Processing for 5G," in Proc. IEEE Globecom Workshops (GC Wkshps), 2015, pp. 1-7.

[30] D. Tse and P. Viswanath, Fundamentals of Wireless Communication. Cambridge University Press, 2005.

[31] D. G. Martinson, Quantitative Methods of Data Analysis for the Physical Sciences and Engineering. Cambridge University Press, 2018

[32] 3GPP, "OFDM Based Flexible Waveform for 5G," 3rd Generation Partnership Project, TR R1-162152, April. 2016.

[33] R. Horst and H. Tuy, Global Optimization: Deterministic Approaches. Springer Science \& Business Media, 2013

[34] W. Yu, G. Ginis, and J. M. Cioffi, "Distributed Multiuser Power Control for Digital Subscriber Lines," IEEE J. Select. Areas Commun., vol. 20 , no. 5, pp. 1105-1115, 2002.

[35] J. Papandriopoulos and J. S. Evans, "Low-Complexity Distributed Algorithms for Spectrum Balancing in Multi-user DSL Networks," in Proc. IEEE International Conference on Communications, vol. 7, 2006, pp. 3270-3275.

[36] M. Grant and S. Boyd, "CVX: Matlab Software for Disciplined Convex Programming, Version 2.1," 2014.

[37] D. Wu, X. Zhang, J. Qiu et al., "A Field Trial of f-OFDM Toward 5G," in 2016 IEEE Globecom Workshops (GC Wkshps), Dec 2016, pp. 1-6.

[38] 3GPP, "Study on Channel Model for Frequencies from 0.5 to $100 \mathrm{GHz}$ (Release 15)," 3rd Generation Partnership Project, TR 38.901, 052017. 